\title{
Eddy-Mean Flow Interaction in the Kuroshio Extension Region
}

\author{
STEPHANIE WATERMAN* \\ Massachusetts Institute of Technology-Woods Hole Oceanographic Institution Joint Program, \\ Woods Hole, Massachusetts \\ NELSON G. HOGG \\ Cornell University, Ithaca, New York \\ STEVEN R. JAYNE \\ Woods Hole Oceanographic Institution, Woods Hole, Massachusetts
}

(Manuscript received 20 August 2010, in final form 17 December 2010)

\begin{abstract}
The authors use data collected by a line of tall current meter moorings deployed across the axis of the Kuroshio Extension (KE) jet at the location of maximum time-mean eddy kinetic energy to characterize the mean jet structure, the eddy variability, and the nature of eddy-mean flow interactions observed during the Kuroshio Extension System Study (KESS). A picture of the 2-yr record mean jet structure is presented in both geographical and stream coordinates, revealing important contrasts in jet strength, width, vertical structure, and flanking recirculation structure. Eddy variability observed is discussed in the context of some of its various sources: jet meandering, rings, waves, and jet instability. Finally, various scenarios for eddy-mean flow interaction consistent with the observations are explored. It is shown that the observed cross-jet distributions of Reynolds stresses at the KESS location are consistent with wave radiation away from the jet, with the sense of the eddy feedback effect on the mean consistent with eddy driving of the observed recirculations. The authors consider these results in the context of a broader description of eddy-mean flow interactions in the larger KE region using KESS data in combination with in situ measurements from past programs in the region and satellite altimetry. This demonstrates important consistencies in the along-stream development of time-mean and eddy properties in the KE with features of an idealized model of a western boundary current (WBC) jet used to understand the nature and importance of eddy-mean flow interactions in WBC jet systems.
\end{abstract}

\section{Introduction}

The Kuroshio Extension System Study (KESS) was a large field experiment focused on the Kuroshio Extension (KE) jet at the location of its maximum time-mean eddy kinetic energy (EKE). Among its goals was to better understand the processes governing the intense meandering and eddy variability of the jet and the nature of the interaction of the jet and its eddy variability (Donohue et al.

\footnotetext{
* Current affiliations: National Oceanography Centre and Grantham Institute for Climate Change, Imperial College London, London, United Kingdom.

Corresponding author address: Stephanie Waterman, National Oceanography Centre, Southampton SO14 3ZH, United Kingdom. E-mail: snw@alum.mit.edu
}

2008). As such, KESS provides new observations of the KE jet, its eddy variability, and their interactions that are unprecedented in both their spatial and temporal resolution. It presents a unique opportunity to improve our understanding of the nature and importance of eddy-mean flow interactions in western boundary current (WBC) jet systems.

A study of the role of eddies and their interaction with the mean flow in WBC jet systems is important because eddy variability likely plays a critical role in WBC jet dynamics. For example, we expect eddies and their nonlinear interactions to impact mean jet-gyre strength, structure, and stability (Thompson 1978; Dewar and Bane 1989; Hogg 1992; Watts et al. 1995); play a role in driving the jet's flanking recirculations (Hogg 1983, 1985, 1993); couple strong upper-ocean motions to deep abyssal motions (Shay et al. 1995; Howden 2000; Watts et al. 2001); and potentially act as a source of the interannual variability observed 


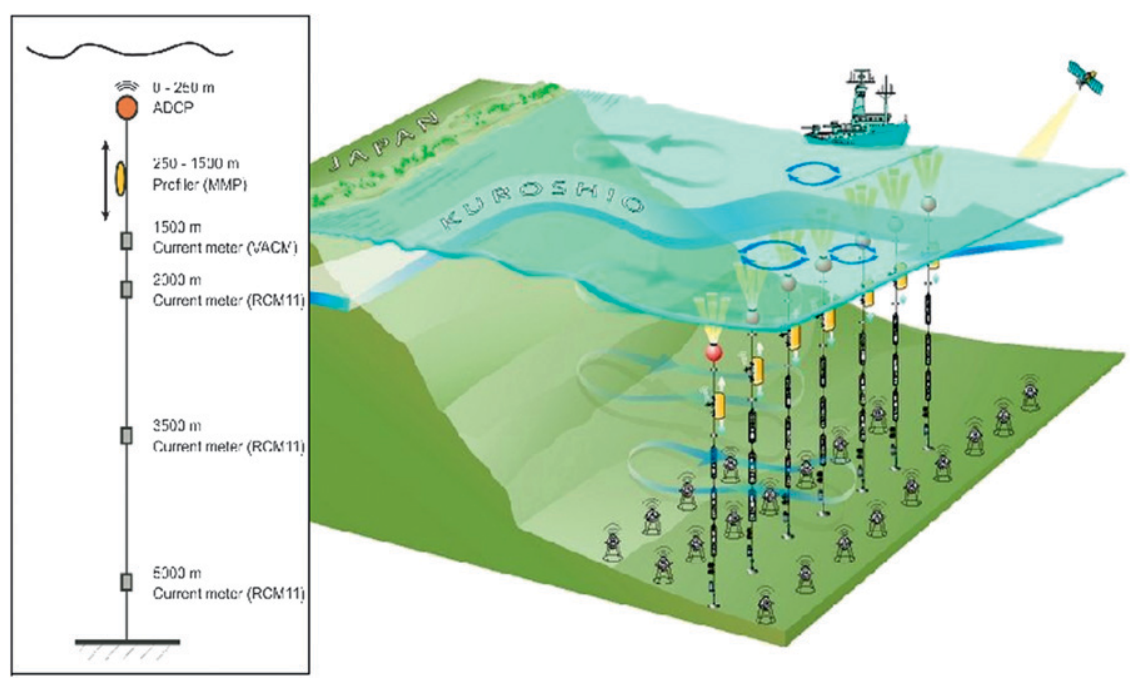

FIG. 1. A schematic of the KESS instrumentation.

in these jet-gyre systems (Spall 1996; Qiu 2000). These effects have important implications for global balances between forcing and dissipation, subtropical-subpolar exchange, the formation of mode water, the storage of heat and potential vorticity (PV), and the steering and intensification of extratropical storms. They are critical to our understanding of the large-scale circulation. Despite this, observational studies on the subject have been rare, largely because of the challenge of obtaining enough observational data to accurately calculate various eddy statistics. Some previous attempts have been made with limited data on regional scales, including Thompson (1978), Hall (1986), Hogg (1993), and Cronin (1996) in the Gulf Stream and Hall (1991), Qiu (1995), and Adamec (1998) in the KE.

We report here on efforts to characterize the mean state, its eddy variability, and their interactions from the KESS mooring data, collected by a line of tall current meter moorings deployed across the axis of the $\mathrm{KE}$ jet and extending into its flanking recirculation gyres. Here and in what follows, we use the word "mean" to describe the time-mean over the 2-yr KESS record. The presentation is organized as follows: In section 2, we provide information about the KESS data as well as other sources of observational data in the region that we use, and we describe the data processing techniques that we employ. In section 3 , we characterize the mean jet structure in both geographical and stream-coordinate frameworks. In section 4 , we consider the nature of the temporal variability observed during the KESS period by describing it in relation to some of its various sources: jet meandering, ring interaction, wave radiation and interaction, and jet stability. In section 5, we explore possible scenarios for eddy-mean flow interactions consistent with the observations. Finally, in section 6, we summarize and discuss the results, as well as provide ideas for future work. Details on linear stability calculations used to assess evidence of jet instability relevant to observations of the KE jet are provided in the appendix.

\section{Methodology}

\section{a. Sources of observational data}

\section{1) KESS DATA}

A four-dimensional, mesoscale-resolving instrument array (Fig. 1) located at the along-stream location of the maximum in EKE (Fig. 2) was deployed in KESS. The observational period spanned a total of 704 days, from

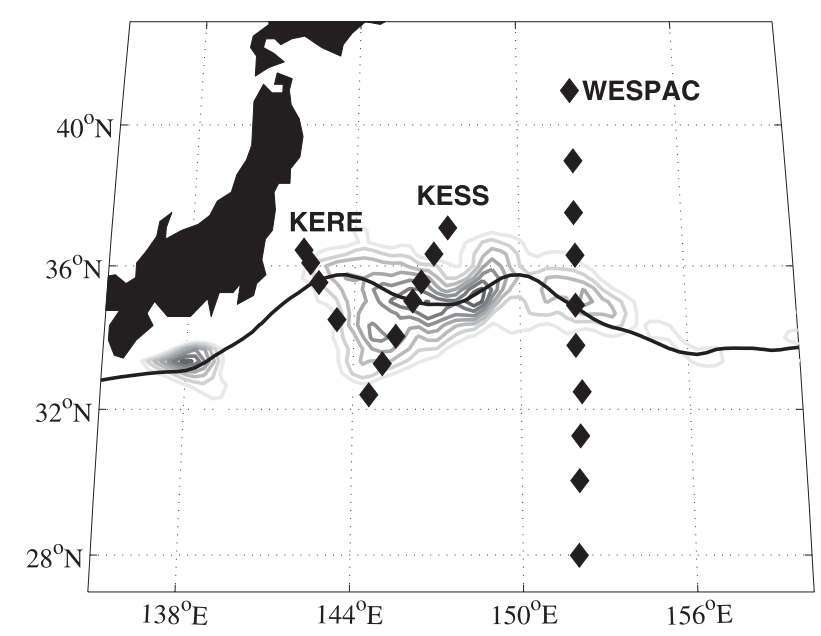

FIG. 2. The location of the KESS, KERE, and WESPAC mooring arrays relative to the mean EKE distribution (contours) in the $\mathrm{KE}$ region. The mean EKE distribution is derived from the 14-yr altimetry record described in the text. 

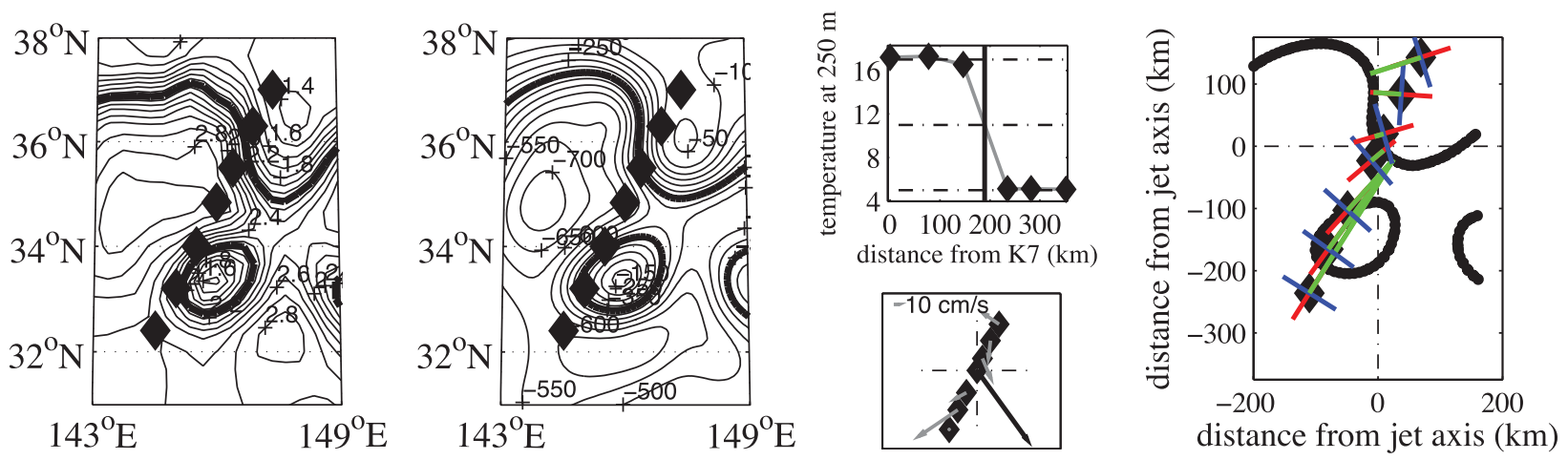

FIG. 3. An illustration of the three different proxies for the jet axis location for the KESS data: (left) the 2.1-m SSH contour (thick black line) in the daily snapshot of SSH measured by satellite altimetry (black contours), (middle left) the 350 -m-depth contour of the $12^{\circ} \mathrm{C}$ isotherm (thick black line) in the daily snapshot of isotherm depth measured by the CPIES (black contours), (middle right) inferring jet axis (top) latitude and (bottom) orientation from (top) the along-mooring-array-line location of the $12^{\circ} \mathrm{C}$ isoterm at 250 - $\mathrm{m}$ depth inferred from the daily subsampled temperature measurements of the mooring ADCPs (black diamonds) and calculated by linear interpolation (line) and (bottom) the orientation of daily subsampled velocity vectors as measured by the mooring ADCPS (vectors), and (right) an illustration of how the stream-coordinate frame is defined at each step. The location and orientation of the jet axis in the vicinity of the mooring array is defined (shown here by the CPIES $12^{\circ} \mathrm{C} 350$-m-depth contour) (thick black line), the distance of each mooring to the closest point on the jet axis is then calculated (green lines), and finally based on the orientation of the jet axis at this closets point, the observed velocities are rotated into a local along-stream (blue) and acrossstream (red) coordinate frame. Diamonds indicate the KESS mooring locations. The example fields shown are from 29 Dec 2004.

spring 2004 until spring 2006. Instrumentation included an array of 7 full-depth moorings (see Jayne et al. 2009), an array of 50 inverted echo sounders equipped with bottom pressure gauges and current meters [current and pressure recording inverted echo sounders (CPIES)] (see Donohue et al. 2010), and a total of 48 profiling Autonomous Profiling Explorer (APEX) floats that were deployed on two occasions within the recirculation gyres (see Qiu et al. 2006, 2008).
In this study, we focus on the analysis of the KESS mooring data. These moorings were deployed across the axis of the KE jet along a northeast-southwest tending line coincident with a Jason-1 satellite altimeter repeat track, spanning its north-south excursions (Fig. 9) and extending into the recirculation gyres. They measured the near-surface velocity field with upward-looking acoustic Doppler current profilers (ADCPs) at 250-m depth; velocity, temperature, and salinity between 250 - and 1500-m
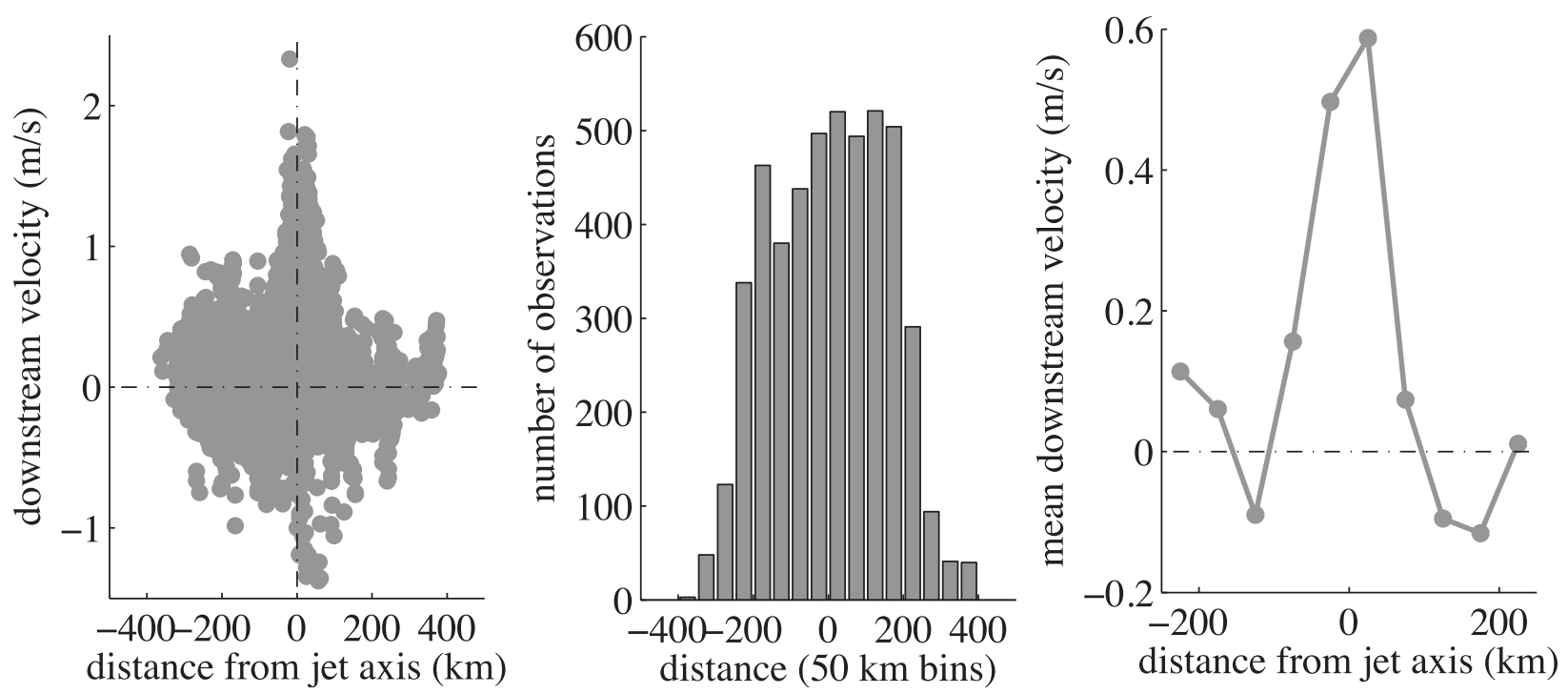

FIG. 4. An illustration of the calculation of the stream-coordinate mean jet structure at 250-m depth. (left) A superposition of all daily measurements of velocity as a function of distance from the instantaneous jet axis, (middle) a histogram illustrating the number of observations in each distance bin for a bin size of $50 \mathrm{~km}$, and (right) the resulting profile of mean along-stream velocity as a function of distance from the jet axis (the "stream-coordinate mean jet") formed from the average velocity measurement in each distance bin. 
depths with McLane Moored Profilers (MMPs) that took a profile once every $15 \mathrm{~h}$; velocity and temperature at 1500-m depth at 30-min intervals with Vector-Averaging Current Meters (VACMs); and finally temperature and velocity at three additional depths beneath the thermocline (2000-, 3500-, and 5000-m depths) at 30-60-min intervals with Aanderaa RCM-11 acoustic current meters. As such, the moorings resolved the fluctuations in the density and velocity fields through most of the water column for time scales from hours to seasons and provided measurements of the flow and temperature fields across the axis of the KE jet with sufficient duration and resolution to investigate mesoscale eddy-mean flow interactions. With the exception of the MMPs, all instruments performed well (for details, see Jayne et al. 2009). Here, we focus on the observations from the ADCPs and current meters and leave the MMP data for a future analysis.

\section{2) OTHER SOURCES OF OBSERVATIONAL DATA IN THE KE REGION}

KESS provided data with high resolution locally, but interest in the along-stream evolution of the jet system motivates us to also look upstream and downstream of KESS. To do this, we exploit additional sources of observational data in the KE region. First, we make use of in situ measurements from past programs in the region: namely, from the "WESPAC" program, located downstream of KESS and conducted in the early 1980s (see Schmitz et al. 1982; Hall 1989), and from the Kuroshio Extension Regional Experiment (KERE) program, located upstream of KESS and conducted in the early 1990s (see Hallock and Teague 1995) (Fig. 2). Both programs consisted of tall mooring arrays with measurements of velocity and density both above and below the thermocline for a duration of $2 \mathrm{yr}$. Second, we make use of the satellite altimetry record in the region, because it provides both a more continuous picture of along-stream development and also a much longer time series to better characterize the time-mean and eddy statistics. The altimeter products were produced by Segment Sol Multimissions d'Alimétrie, d'Orbitographie et de Localization Precise/Data Unification and Altimeter Combination System (Ssalto/Duacs) and distributed by Archiving, Validation, and Interpretation of Satellite Oceanographic data (AVISO). The 14-yr record we refer to is from the beginning of the AVISO altimeter dataset (1992) until the end of the KESS period (spring 2006).

\section{b. Data processing and calculation of $a$ stream-coordinate frame}

KESS mooring velocity records used in the study were low-pass filtered and subsampled at one day. ADCP records were corrected for mooring motion. RCM-11 speeds

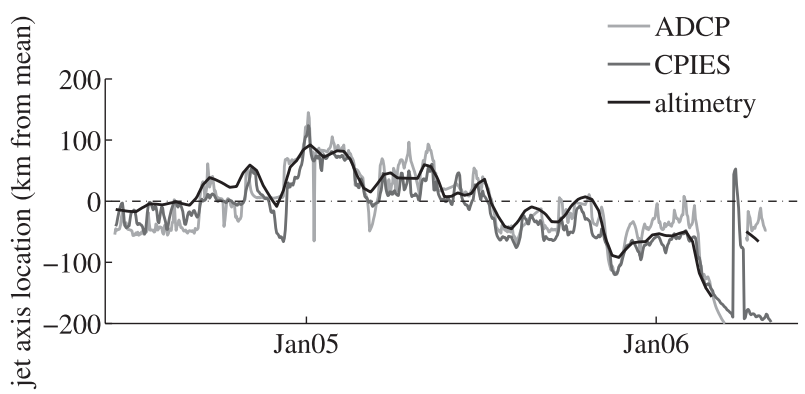

FIG. 5. The time series of jet axis location along the KESS mooring line for each of the three proxies for jet axis position considered in the definition of the stream-coordinate system.

were adjusted upward by $10 \%$ to account for a bias in their measurement compared to other current meters (Hogg and Frye 2007). Small gaps in the record were filled with the value of the record mean in the case of the ADCP measurements and by a gap-filling procedure that used the records at other subthermocline depths plus the vertical structure of the first empirical orthogonal function (EOF) to reconstruct missing record values at deep levels in the case of the VACM and RCM-11 records. For processing details of the WESPAC and KERE records, refer to Schmitz et al. (1982) and Hallock and Teague (1995), respectively.

Given the large "smearing" effect of jet meandering on mean jet structure, it is useful to compute a streamcoordinate frame for the jet for all sets of observations. This frame is defined by a time-varying origin and alongstream and cross-stream axes orientation based on the instantaneous position and orientation of the jet axis in the vicinity of the array. The calculation consists of the following steps: At each time step we 1) define the location and orientation of the jet axis in the vicinity of the array;2) calculate the distance of each mooring to the closest point on the jet axis and the orientation of the jet axis there; and 3) rotate the observed velocities based on that orientation into along-stream and cross-stream components (Fig. 3). Daily values of distance and velocity are then binned according to distance from the jet axis and averaged in each bin. A bin size of 50-km width is chosen to ensure a reasonable number of observations $(>200)$ in each bin within $\pm 200 \mathrm{~km}$ from the jet axis (Fig. 4). The result of the procedure is a profile of mean along-stream and crossstream velocity as a function of distance from the jet axis.

In the case of the KESS data, the stream-coordinate calculation is done using three different independent proxies for the jet axis location: 1) the 2.1-m sea surface height ( $\mathrm{SSH}$ ) contour measured by satellite altimetry; 2 ) a proxy for thermocline depth (the 350-m-depth contour of the $12^{\circ} \mathrm{C}$ isotherm) measured by the CPIES; and 3) the latitude where the $11^{\circ} \mathrm{C}$ isotherm is at 250 -m depth using 

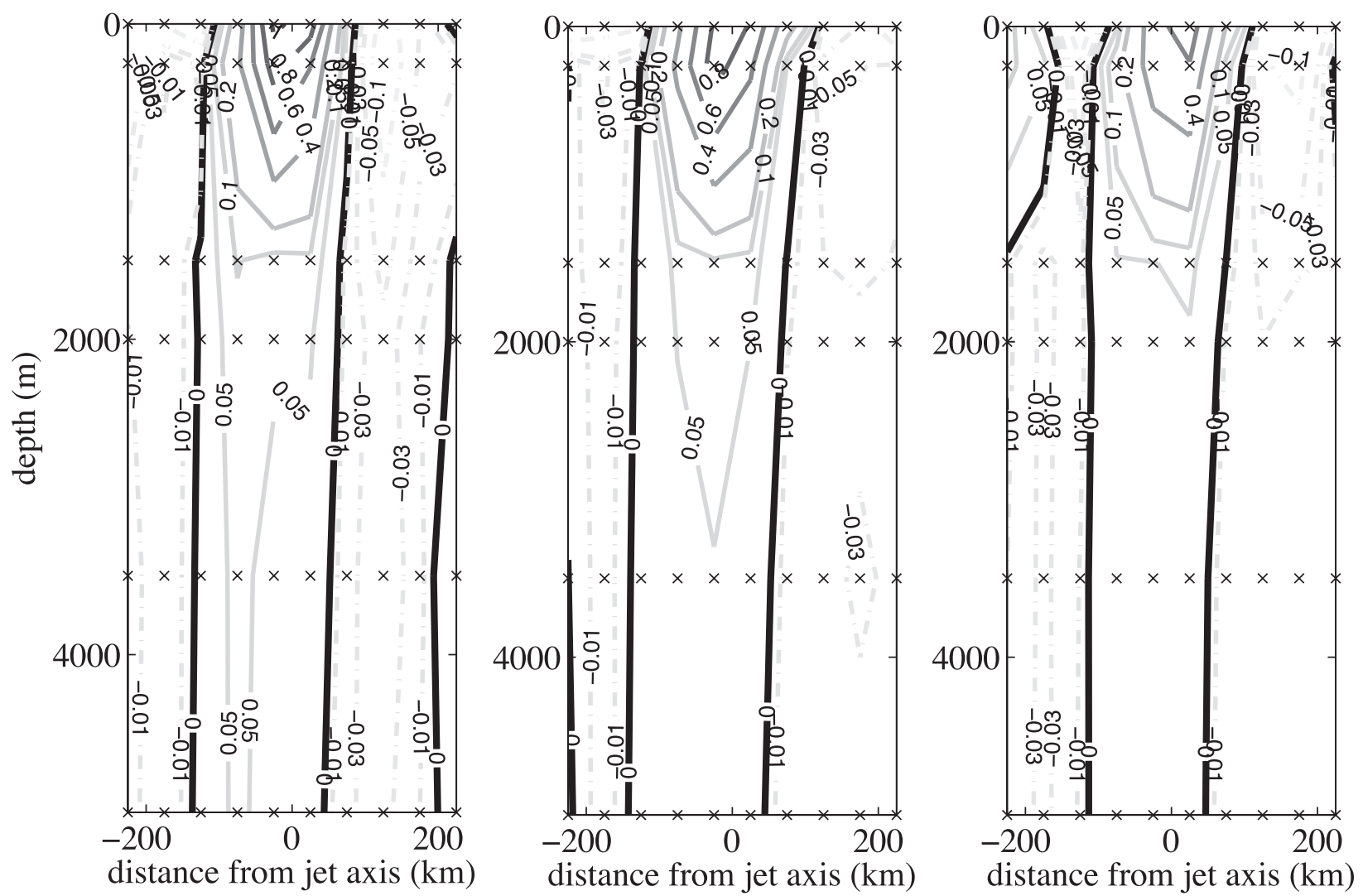

FIG. 6. A comparison of the stream-coordinate descriptions of the mean jet structure computed using each of the three proxies for jet axis position for the KESS data: (left) the $2.1-\mathrm{m} \mathrm{SSH}$ contour measured by altimetry, (middle) the 350 - $\mathrm{m}$ depth contour of the $12^{\circ} \mathrm{C}$ isotherm measured by the CPIES, and (right) temperature and velocity measurements at 250-m depth measured by the mooring ADCPs.

the mooring array ADCP measurements and calculated by linear interpolation (Fig. 3). In the case of the latter, because no information about the orientation of the jet axis is given by this proxy, it is taken to be the orientation of the velocity vector with the largest eastward zonal component measured by the array on that day. All three proxies give very consistent results for both the jet axis position (Fig. 5) and the stream-coordinate structure derived from it, including the existence of westward recirculations to both the north and south of the mean jet (Fig. 6). This gives confidence that each is a useful way to determine the jet axis position. A quantitative comparison of the three descriptions is given in Table 1. It is not surprising that the proxy based on altimetry data gives the maximum jet speed, because this proxy will be best aligned with the altimetry-derived surface flow where the largest velocities are recorded. Reduced meandering extent and activity in this description may be an artifact of the reduced spatial resolution of this proxy. ${ }^{1}$ Finally,

\footnotetext{
${ }^{1}$ To define the jet orientation in the vicinity of the array, it is necessary to use the AVISO gridded product with $1 / 3^{\circ}$ resolution and objectively mapped using a $\sim 300-\mathrm{km}$ length scale.
}

we note that the jet defined using the ADCP data has significantly weaker velocity magnitudes and net transport than the jets defined using the two other proxies, likely a consequence of the more coarsely resolved and crude definition of the jet's orientation. We use the

TABLE 1. A comparison of the three stream-coordinate descriptions of the KE jet from KESS data.

\begin{tabular}{lccr}
\hline \hline & Altimetry & CPIES & ADCP \\
\hline Max instantaneous & 1.9 & 1.9 & 1.4 \\
$\quad$ speed $\left(\mathrm{m} \mathrm{s}^{-1}\right)$ & & & \\
Peak mean jet speed $\left(\mathrm{m} \mathrm{s}^{-1}\right)$ & 1.5 & 1.1 & 0.9 \\
Depth-averaged mean jet & 191 & 207 & 180 \\
$\quad$ width $(\mathrm{km})$ & 114 & 114 & 80 \\
Mean jet transport & & & \\
$\quad\left[\right.$ Sv $\left.\left(1 \mathrm{~Sv} \equiv 1^{6} \mathrm{~m}^{3} \mathrm{~s}^{-1}\right)\right]$ & 15 & 11 & 15 \\
Mean recirculation transport* $(\mathrm{Sv})$ & 3 & 2 & 5 \\
$\quad$ Northern recirculation gyre & & & \\
$\quad$ Southern recirculation gyre & 249 & 322 & 347 \\
Jet meandering extent $(\mathrm{km})$ & 49 & 64 & 57 \\
Jet axis position std dev $(\mathrm{km})$ & & &
\end{tabular}

* Recirculation transport is likely to be only a partial measure of the total recirculation transport, given that the mooring array did not extend through the full extent of the recirculation gyres. 

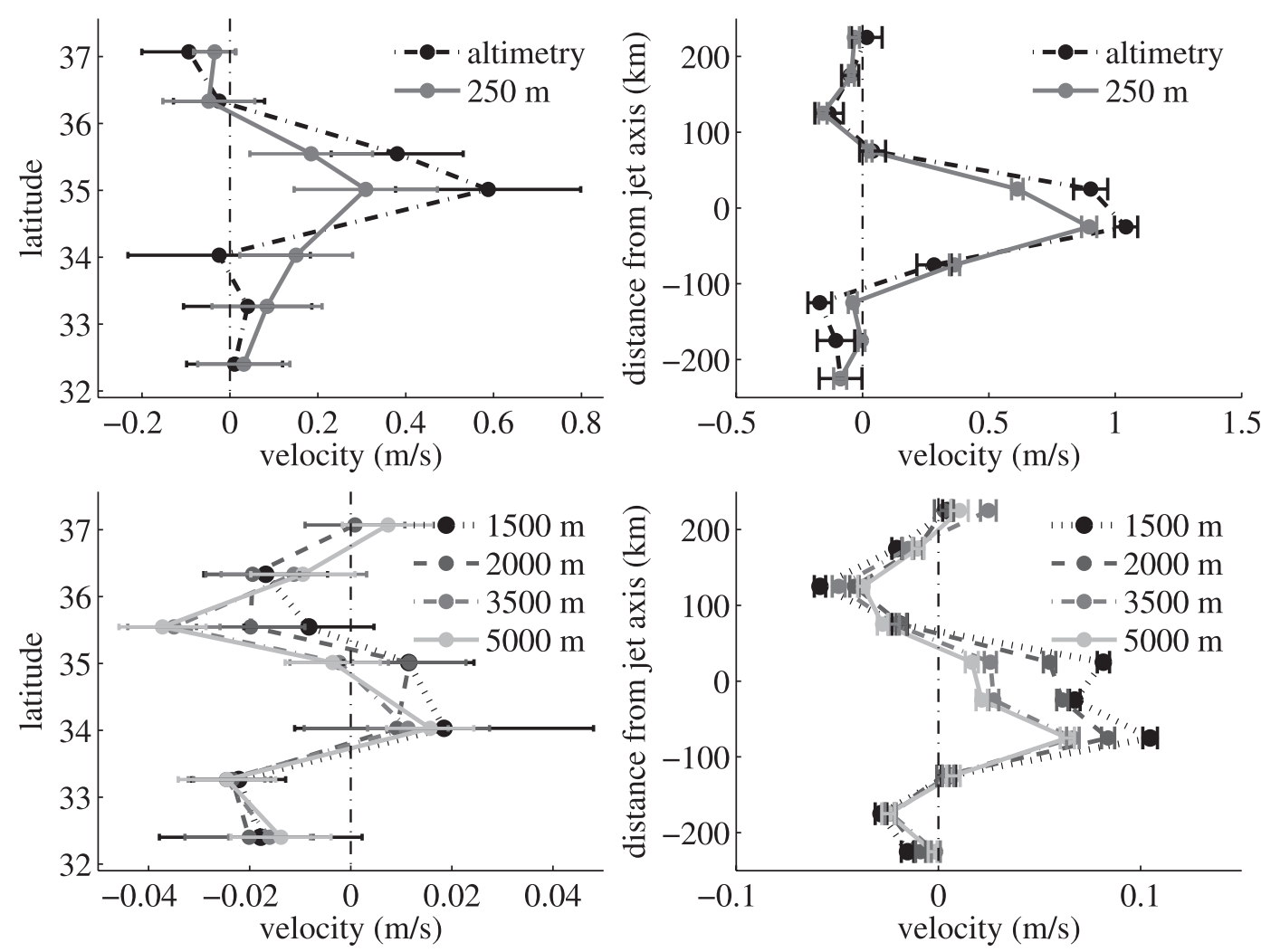

FIG. 7. Cross-jet profiles of (left) the mean zonal velocity and (right) the along-stream component of velocity in the stream-coordinate frame at (top to bottom) six levels in the vertical.

CPIES data derived description in what follows in cases where a single stream-coordinate description is discussed. It is reasonable to have the most confidence in this description, because it describes the position and orientation of the jet axis in the vicinity of the KESS array with the highest spatial and temporal resolution.

A stream-coordinate mean system is also defined for the KERE and WESPAC array mooring measurements. Here, neither altimetry data nor CPIES data are available, so the position of the jet axis is taken to be that given by the latitude of the $6^{\circ} \mathrm{C}$ isotherm at $500-\mathrm{m}$ depth using the same method applied to the KESS ADCP data.

Finally, a stream-coordinate mean picture of the KE jet structure is computed using the altimetry fields from the 14-yr record. As in the calculation for the KESS mooring array observations, the stream-coordinate mean is computed by defining the jet axis by the 2.1-m SSH contour and then computing the distance to the nearest point on the jet axis and rotating the velocities at each grid point. Velocities are then binned and averaged based on distance from the jet axis at each longitudinal grid point. This produces a series of meridional profiles of the mean stream-coordinate jet structure at each $1 / 3^{\circ}$ longitude.

\section{Mean jet structure}

As a first step in characterizing eddy-mean flow interactions during KESS, we describe the mean jet structure measured by the KESS mooring array computed over the 704-day KESS period (15 June 2004-19 May 2006). Here, we provide a brief summary, because the KESS mean jet structure is well documented in Jayne et al. (2009).

\section{a. Geographical description}

Computing the mean value of the horizontal velocity as a function of latitude and depth reveals a strong, surfaceintensified jet oriented to the southeast (Jayne et al. 2009). Satellite altimetry measurements indicate a mean (geostrophic) jet at the surface at this location with a peak mean velocity of $\sim 0.6 \mathrm{~m} \mathrm{~s}^{-1}$ and a width of $\sim 2.3^{\circ}$ latitude $(\sim 250 \mathrm{~km}) .^{2}$ KESS subsurface measurements in the upper ocean indicate a sharp decay in mean jet strength with depth (the mean peak velocity is reduced to $0.3 \mathrm{~m} \mathrm{~s}^{-1}$ at 250-m depth and $0.02 \mathrm{~m} \mathrm{~s}^{-1}$ at $1500-\mathrm{m} \mathrm{depth}$ ) (Fig. 7,

\footnotetext{
${ }^{2}$ Note that altimetry gives a smoothed description of the jet because of the $\sim 300-\mathrm{km}$ length scale used in the objective mapping.
} 


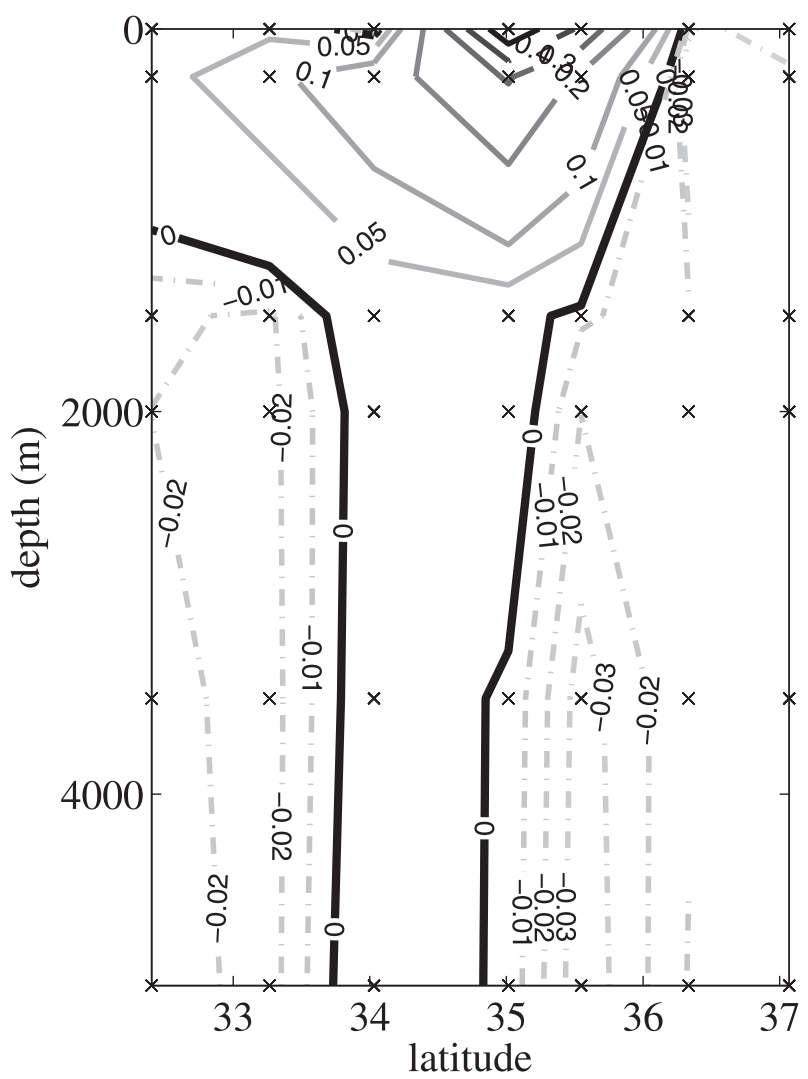

FIG. 8. A contour plot of mean zonal velocity as a function of latitude and depth summarizing the geographical description of the mean jet structure.

left), as well as a shift of the jet axis to the south, consistent with thermal wind balance (Fig. 8). In contrast, in the deep ocean, the jet strength and structure show very little depth dependence (Fig. 7, bottom left). The deep jet structure also shows weakly depth-dependent westward flows on both the northern and southern flanks of the mean jet with velocity magnitudes comparable to that of the deep jet itself. Flanking flows are weak and disorganized in the upper ocean (there is no clear evidence of westward recirculations), with weak westward flows observed to the north of the jet but not to the south (Fig. 8).

\section{b. Stream-coordinate description}

The stream-coordinate view of mean jet structure at the KESS location is summarized in Figs. 6 and 7 and Table 1. As in the geographical mean picture, the streamcoordinate mean jet is a strong, sharp, surface-intensified jet oriented to the southeast that extends throughout the water column. As one would expect, it is stronger and sharper than its geographical mean counterpart (the mean peak surface velocity is $\sim 1.0 \mathrm{~m} \mathrm{~s}^{-1}$ and the mean surface width is $\sim 180 \mathrm{~km}$ ). The mean subsurface upper-ocean jet is also significantly stronger (with strengths of $0.9 \mathrm{~m} \mathrm{~s}^{-1}$ at 250-m depth and $\sim 0.1 \mathrm{~m} \mathrm{~s}^{-1}$ at $1500-\mathrm{m}$ depth). Thus, in this view, the mean jet is much more strongly sheared in both the horizontal and vertical than it is in the geographical frame. This has important implications for its stability properties as we will discuss in section 3 . The stream-coordinate view of mean jet structure shows westward flanking flows in the upper ocean as well as in the deep ocean, suggesting the existence of weakly depth-dependent recirculations throughout the water column (Fig. 6). These recirculations are much more barotropic than the strongly baroclinic jet, with speeds of similar magnitude in both the upper and deep ocean. Their absence in the upper ocean in the geographical mean picture is a result of the meandering of the strong upper-ocean jet smearing out the relatively weak westward flanking flows. Prior to KESS, although the presence of a recirculation gyre to the south of the jet had been documented, the existence of a northern recirculation gyre was in question. The existence of both a northern and southern recirculation gyre is a robust result of the analysis here as well as the analysis of KESS data of other forms (see Jayne et al. 2009).

\section{Eddy variability}

Next we consider the nature of the temporal variability observed during KESS by describing it in relation to some of its various sources: jet meandering, ring interaction, wave radiation/interaction, and jet instability.

\section{a. Jet meandering}

We define jet meandering as the northeast-southwest migration of the jet's position along the mooring line. The meandering of the jet results in the intermittent presence and absence of the jet at a given mooring, and we expect this to be a dominant source of the variability observed there. Here, we describe the time series of the jet axis position along the array to characterize the jet's meandering. We also compare the descriptions of the mean jet structure and its variability in a geographical frame versus the stream-coordinate description. The latter, being centered on the instantaneous jet axis, largely removes the effects of jet meandering and, as such, differences between these two descriptions can be attributed to jet meandering effects.

As discussed in section $2 \mathrm{~b}$, the KESS dataset provides various proxies for the jet axis position, both measured in situ and sensed remotely, and these data give information about the nature and the extent of the meandering of the jet at the KESS location. A superposition of snapshots of the jet path during the KESS period (Fig. 9) illustrates that the extent of the jet's north-south meandering at the KESS location spans several degrees of latitude and that, during the KESS period, the jet traversed the mooring 

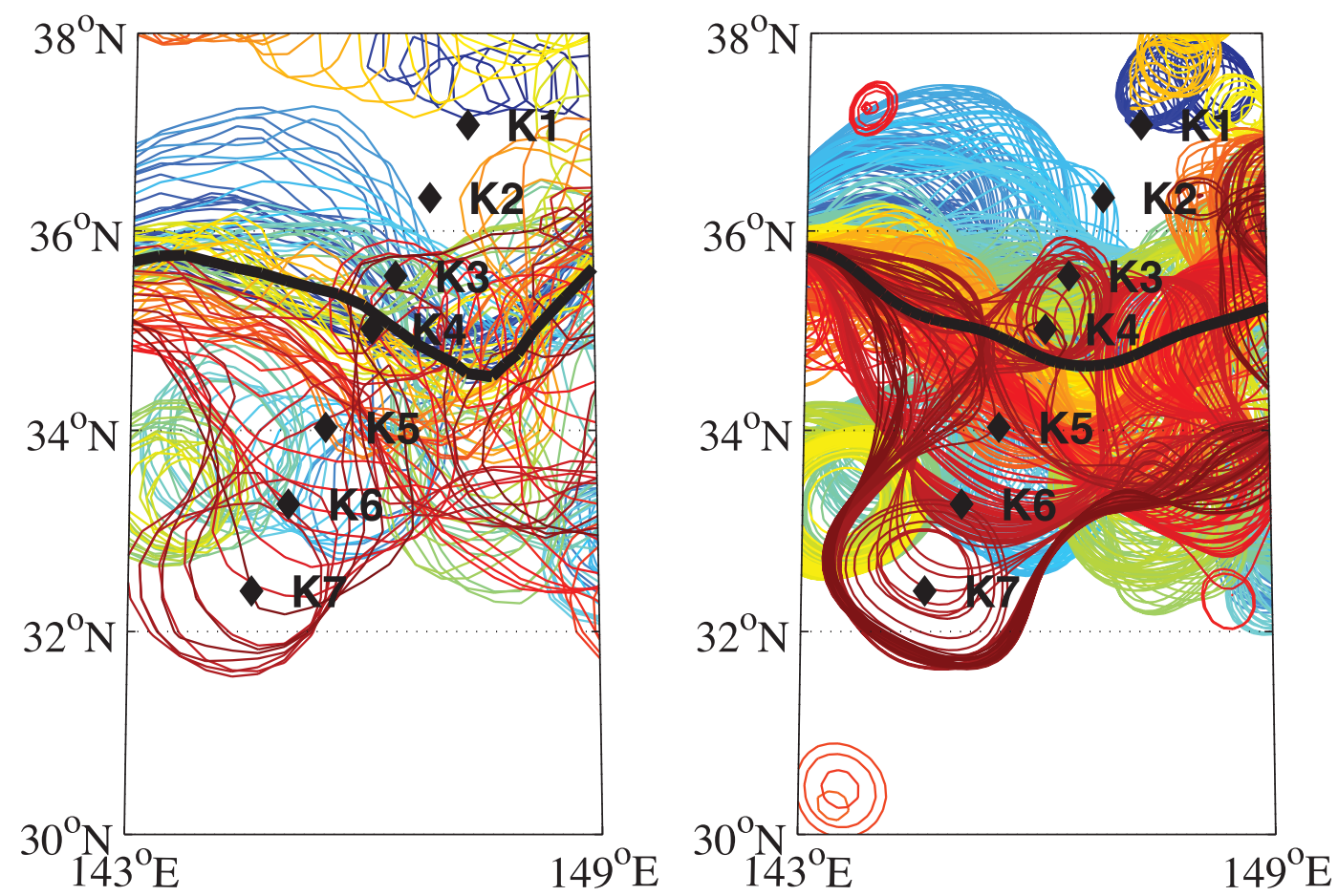

FIG. 9. A superposition of (left) weekly snapshots of the 2.1-m SSH contour measured by satellite altimetry and (right) daily snapshots of the thermocline depth from KESS CPIES data, each serving as a proxy for the jet axis position.

array as far south as south of the southernmost mooring (K7) and as far north as the K2 mooring. The time series of jet axis position (Fig. 5) gives quantitative information about the meandering: during the KESS period, the jet axis location varied about its mean position with a standard deviation on the order of $60 \mathrm{~km}$ and a maximum range on the order of $300 \mathrm{~km}$. Spectra of the time series of jet axis position (Fig. 10) indicate enhanced energy at a 50-day period and in a broader band centered around a 23-day period. This second frequency is consistent with the time scales predicted by appropriate linear stability calculations, which are on the order of 20-30 days for the fastest growing modes. See the appendix for details.

Comparing the geographical versus stream-coordinate descriptions of the cross-jet distributions of mean velocity and EKE give indications of the effect of jet meandering on the mean jet structure and its variability (Fig. 11). Meandering reduces the peak mean jet speed (by $\sim 50 \%$ in the upper ocean and $\sim 75 \%$ in the deep ocean) and eliminates the recirculations in the upper layer in the geographical mean, a consequence presumably of occasional strong eastward velocities associated with the meandering jet dominating over weak flanking westward flows in the computation of the time average. Note that the differences in the geographical versus stream-coordinate mean structures, particularly with respect to the existence of mean westward recirculations, are much less significant in the deep ocean than in the upper ocean, consistent with the fact that the jet is much stronger near the surface. The comparison of the cross-jet EKE distributions (Fig. 11, right) reveals structure in the stream-coordinate description that is hidden by the meandering in the geographical mean picture. In particular, in the upper ocean the stream-coordinate EKE distribution is sharply peaked at the jet axis and has local minima inside the recirculations, a structure not seen in the geographical distribution. In the deep ocean in contrast, both EKE distributions are similar, suggesting that meandering has less influence in eroding structure there. In both the upper and deep ocean, the higher EKE levels in the geographical frame reflect the additional variability due to jet meandering.

Finally, comparing the cross-jet integrated EKE levels for the geographical versus stream-coordinate frames over a common cross-jet distance gives a measure of the relative importance of meandering in the total variability. EKE levels in the stream-coordinate frame are approximately $80 \%$ of that in the geographical frame, suggesting jet meandering accounts for approximately $20 \%$ of the jet's total variability. Surprisingly, this fraction is common to both the upper ocean (where the integrated EKE in the stream-coordinate frame is $78 \%$ of that in the geographical frame) and the deep ocean (where it is $80 \%$ of the geographical frame value). The relative importance 


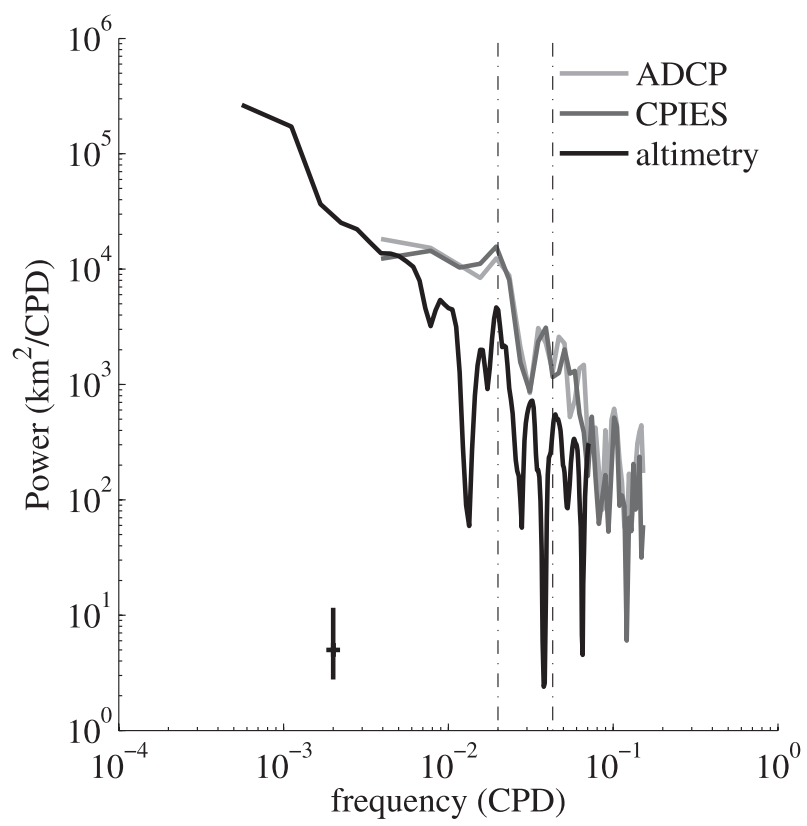

FIG. 10. An estimate of the power spectral density computed from the time series of jet axis position for each of the three proxies for jet axis location. Dash-dotted lines indicate periods of 50 and 23 days. The height of the cross in the lower left indicates the $95 \%$ confidence interval.

of meandering in the upper ocean, where the surfaceintensified jet is located is perhaps offset by rings, wind, and other sources of variability that also play a significant role in the upper ocean.

\section{b. Ring interactions}

We define ring interactions as coherent ring or eddy-like structures with anomalous temperature signatures on the flanks of the jet intercepting the mooring array. In the KESS period, SSH fields from altimetry show rings both pinching off from the jet in the vicinity of the array as well as propagating into the mooring area from the east. The presence of warm and cold core rings at the KESS moorings were identified by examining the cross-jet profiles of temperature as a function of distance from the jet axis and flagging large deviations from the mean temperature on the jet flanks. Specifically, at each day, the daily subsampled ADCP temperature measurements at $250-\mathrm{m}$ depth at $\pm 85 \mathrm{~km}$ and beyond from the jet axis were compared to the record mean temperature on the northern flank (the mean of all temperature measurements beyond $+85 \mathrm{~km}$ from the jet axis) and the southern flank (the mean of all temperature measurements beyond $-85 \mathrm{~km}$ from the jet axis) as appropriate. If any of these daily temperature measurements deviated from the mean by more than one standard deviation, the day was flagged. These times were then cross-checked by confirming the presence of rings in altimetry snapshots of sea surface height.

We find that a ring was present in the vicinity of the KESS array for 187 days out of the 704-day-long record (i.e., $\sim 25 \%$ of the time). These interactions were not uniformly distributed in time but rather were more frequent in the winter of 2005 and in the winter-spring of 2006 (Fig. 12 , left). Examination of the full altimetry record indicates that this wintertime elevated level is not generally the case. Rings passed by all moorings both to the north and south of the jet (Fig. 12, right). The largest number of ring occurrences was at the moorings $\mathrm{K} 5$ and $\mathrm{K} 6$ (almost always south of the jet), where the largest and strongest rings (as seen in the altimetry record) tend to pass.

Comparing the mean jet structure and EKE distributions computed from the full time series versus the time series with ring events removed (Fig. 13) gives indication of the effect of rings on the mean jet structure and its variability. Interestingly, the effect of rings on the flanking mean jet structure is negligible (Fig. 13, left). Westward recirculations remain a feature whether or not ring events are included, implying that their existence is not simply a function of the time averaging of rings propagating westward on the flanks of the jet. In contrast, including ring interactions increases the peak values in EKE on the outer edges of the recirculations $( \pm 200 \mathrm{~km}$ from the mean jet axis) by approximately $50 \%$. In short, ring interactions contribute significantly to the variance structure on the flanks of the jet but not to the mean jet structure there, assuming that their influence exists only during the times when they are present (rings influencing the structure of the jet in such a way that dynamical changes result from their passage is of course plausible and not taken into account by this simple test). Most importantly, with or without rings included, the mean recirculations remain.

Comparing the integrated EKE level on each flank of the jet for the full time series versus the time series with the rings events removed shows that rings account for $38 \%$ of the total variability on the jet flanks in the upper ocean and $28 \%$ in the deep ocean.

\section{c. Wave radiation and interaction}

We define waves as neutral periodic and propagating disturbances. There is the potential for waves to exist in the system, either being radiated from the jet, generated by jet instability, or forced remotely and interacting with the jet-gyre system. Indeed, a striking feature seen in animations of the instantaneous velocity vectors measured by the KESS mooring array is the periodic rotation of the velocity vectors on the flanks of the jet, suggestive of wave activity there. In practice, it is difficult to distinguish between neutral wave motions and exponentially growing disturbances as generated by instability. Here, we examine 

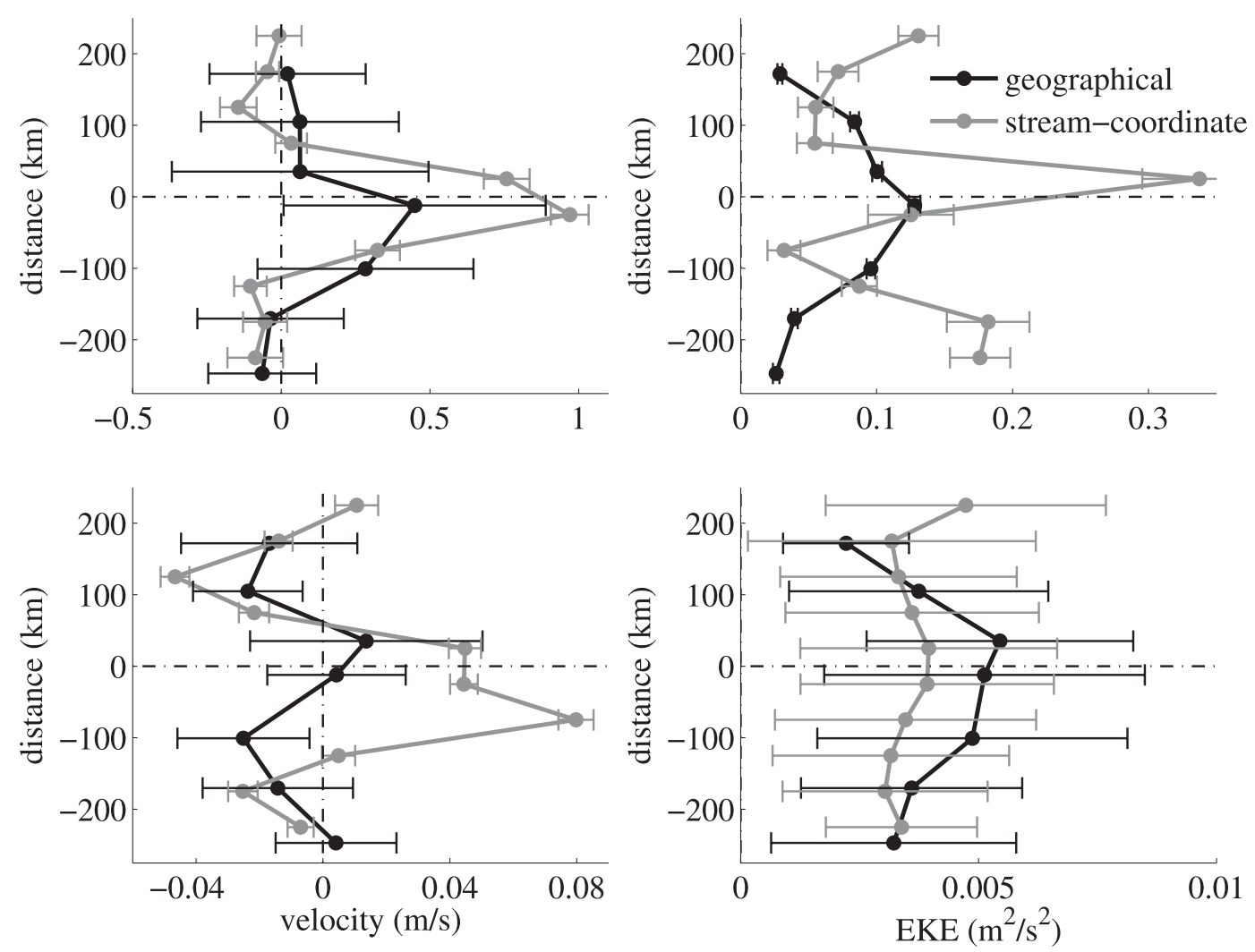

FIG. 11. A comparison of the cross-jet distributions of (left) mean zonal-along-stream velocity and (right) EKE for the geographical and stream-coordinate frames. (top) The upper-ocean average (the average of the surface profile derived from altimetry and the profile at 250-m depth) and (bottom) the deep-ocean average (the average of the profiles at 1500-, 2000-, 3500- and 5000-m depth). Here, and in all following relevant figures, error bars indicate the standard error in the mean assuming a number of degrees of freedom given by the number of decorrelation time scales contained in the record length in the case of the geographical mean and the number of observations forming the average in each distance bin in the case of the stream-coordinate mean.

the observed variability for propagating signals and test the consistency of a linear dispersion relation with the observed frequency and phase propagation for mesoscale frequencies showing enhanced energy in the system.

Hovmöller diagrams formed from the velocity time series show two periods of distinct wave-like propagation (Fig. 14): in the winter of 2005 and in the spring of 2006. It is interesting to note that these times roughly correspond to times of elevated ring activity in the upper ocean (Fig. 12), preceding times of enhanced ring activity slightly. These propagating signals are seen at all depths but are most distinct at the abyssal levels, likely because of the less noisy character of the fields there, which makes the wave signals easier to see.

Spectra of the mooring velocity records show enhanced energy at a number of different mesoscale frequencies that could indicate neutral wave motions or jet instability. In general, there are peaks in power near the 100-day period (most predominantly in the deep ocean), near the 40-day period, and in the 10-20-day-period range (Fig. 15).
The highest of these frequencies is suggestive of a jet instability time scale (see the appendix), but energy at lower frequencies may be associated with Rossby waves either radiated from the jet or generated remotely. Support for the classification of 40-day-period motions as Rossby waves is provided by Fig. 16, which demonstrates that the observed wavenumber associated with this wave activity is consistent with the barotropic Rossby wave dispersion relation on the sloped topography of the KESS region. The linear variation of phase with latitude (Fig. 16, left) implies a constant cross-jet wavenumber given by the slope. The intersection of the circle \{representing all possible pairs of $k-l$ wavenumbers for a barotropic Rossby wave of the 40-day period on the sloped topography in the KESS region [see Greene et al. (2010) for the full formulation] , and the line (corresponding to pairs of $k-l$ wavenumbers consistent with the observed cross jet wavenumber; Fig. 16, right) implies the observed phase variation with latitude is consistent with a barotropic topographic Rossby wave. Analysis of these features using 

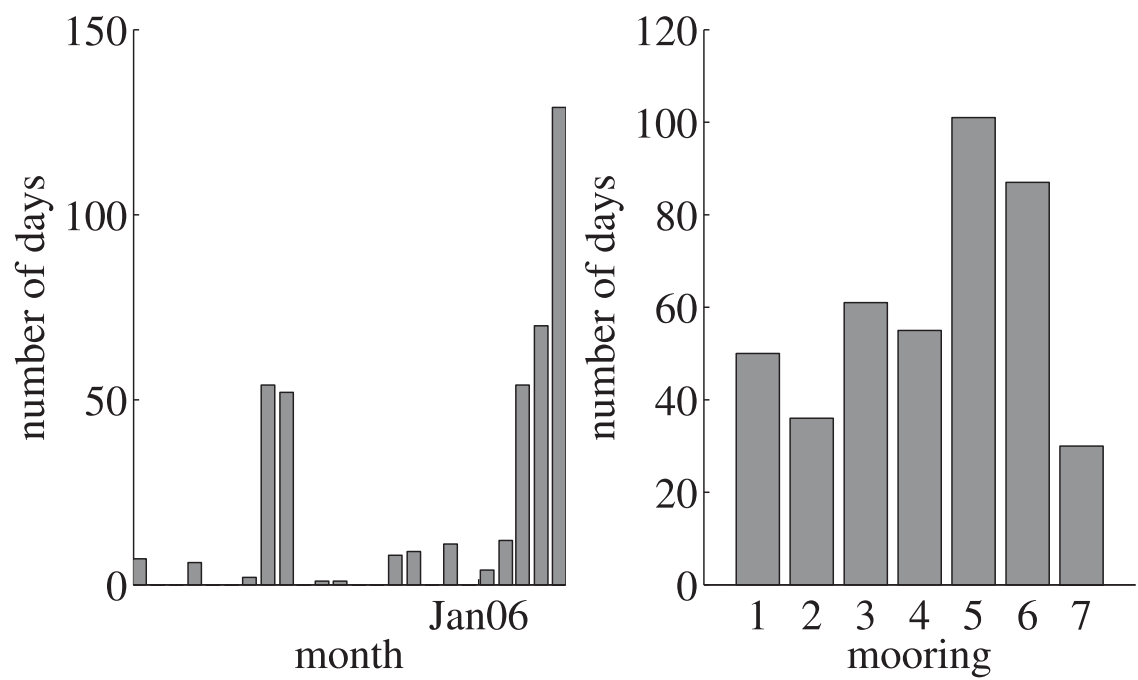

FIG. 12. Histograms showing the distribution of ring events in (left) time and (right) space.

the KESS CPIES data similarly identifies them as barotropic topographic Rossby waves. See Greene (2010) for a full discussion.

We can get an indication of the relative importance of this wave at 40-day period to the total variance by noting that it is described by the first complex empirical orthogonal function (CEOF) mode that accounts for $51 \%$ of the total variance at this time scale, and that the 40-day period peak in the spectra accounts for approximately $18 \%$ of the total variance at all time scales. Hence, we
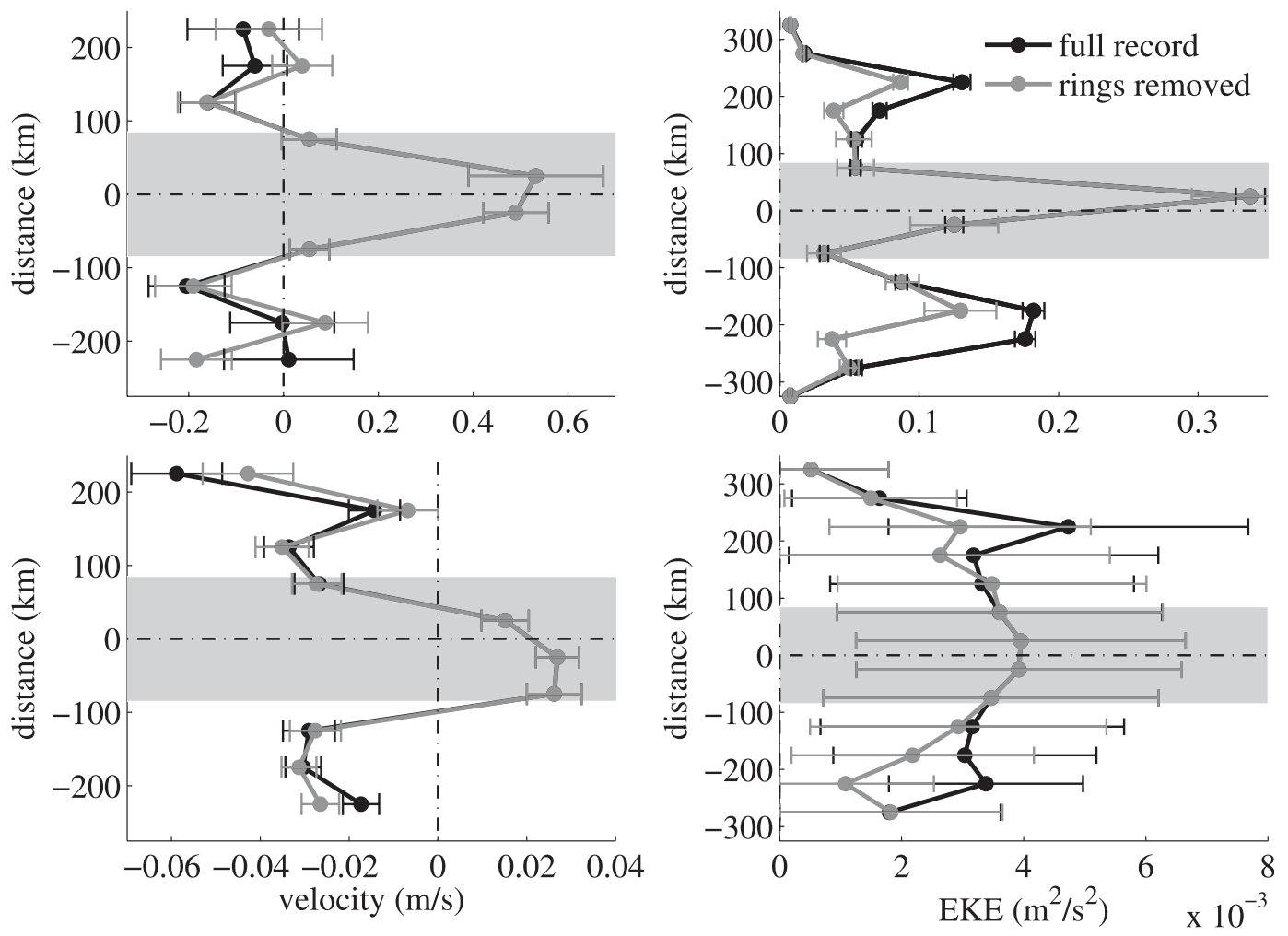

FIG. 13. A comparison of the cross-jet distributions of (left) mean along-stream velocity and (right) EKE computed from the full time series vs the time series with times corresponding to when rings interacted with the mooring array removed. (top) The upper-ocean average and (bottom) the deep-ocean average (as defined in the caption of Fig. 11) are shown. 


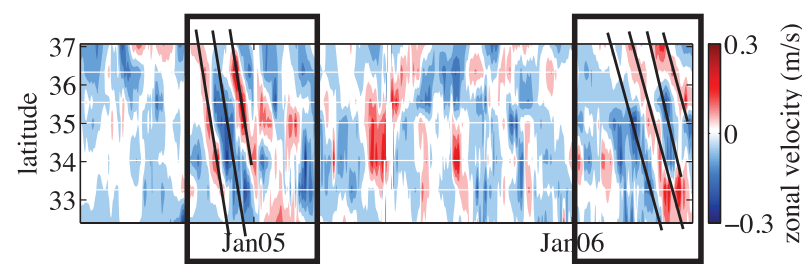

FIG. 14. A Hovmöller plot of zonal velocity at 5000-m depth.

estimate this wave motion to account for approximately $10 \%$ of the variance in the geographical frame.

\section{d. Jet instability}

Finally, we define instability here as variability arising from the hydrodynamic instability of the horizontal and vertical shears of the jet. This may include trapped modes in which the amplitude of the variability decays with distance from the jet and may also include radiating modes, which are capable of propagating to the far field. Again, it is difficult to definitively distinguish neutral wave variability from variability sourced in jet instability. Here, we look for enhanced energy at expected jet instability time scales and consider observed velocity shears in the context of stability criteria to evaluate the KE jet's stability properties.

As discussed in section $4 \mathrm{c}$, spectra of the mooring velocity records show enhanced energy at a number of different mesoscale frequencies (Fig. 15). The frequency band corresponding to periods on the order of 20 days is consistent with the time scale of barotropic instability of the $\mathrm{KE}$ jet as predicted by a linear stability calculation (see the appendix for details), and thus enhanced energy at this time scale provides support for the hypothesis that some of the variability in the KE originates from the instability of the KE jet.

Insight into the potential stability properties of the jet is further gained by considering the observed velocity shears in the context of necessary conditions for instability. The relevant shears to consider are not those associated with the mean jet but rather the instantaneous jet structure. Hence, to more accurately evaluate the stability properties of the jet, we examine both the shears associated with the mean stream-coordinate jet structure (to be thought of as a mean or typical snapshot of instantaneous jet structure, called the synoptic mean here) and extreme instantaneous values in the time series. These horizontal and vertical shears and their associated implications for the stability properties of the jet are given in Figs. 17 and 18 .

One way to get an indication of the jet's potential for barotropic and baroclinic instability is to apply the idealized Rayleigh and Phillips model necessary conditions for instability to the observed horizontal and vertical shears separately. Strictly, one cannot isolate these instability criteria, as the necessary condition for instability of a flow with both horizontal and vertical shear is defined by a change of sign of its total PV gradient. Nevertheless, there is heuristic value in considering the potential for barotropic and baroclinic instability independently to gain insight into the relative contributions of horizontal and vertical shears. This is considered in Fig. 17.

To evaluate the magnitude of the horizontal shear in the context of its potential for barotropic instability, we compute the cross-jet distribution of the meridional gradient of the barotropic PV, $Q_{y}=\beta-U_{y y}$. Here, $\beta$ is the meridional gradient of the planetary vorticity and $U_{y y}$ is the meridional gradient of the jet's meridional shear $U_{y}$, which approximates the jet's relative vorticity. The Rayleigh necessary condition for barotropic instability requires this quantity change sign in the horizontal, which, as is shown, is satisfied in both the synoptic mean and extreme snapshot.

To evaluate the magnitude of the vertical shear in the context of its potential for baroclinic instability, we approximate the vertical structure of the system as consisting of two layers and consider the vertical shear between them, $\Delta U=U_{1}-U_{2}$, relative to the critical value given by the Phillips model, $\Delta U_{\text {critical }}=\beta_{\text {nd }} / F_{2}{ }^{3}$ For layer velocities, we take the upper-ocean mean (the average of surface and 250-m-depth values) and the deep-ocean mean (the average of 1500-, 2000-, 3500-, and 5000-m-depth values) of the peak jet velocity at the jet axis but note that this is an approximation as the stability criterion is derived for layer velocities that are independent of latitude. Here again, the observed vertical shears $\left[\Delta U=0.84 \mathrm{~m} \mathrm{~s}^{-1}\right.$ (synoptic mean) and $\Delta U=1.02 \mathrm{~m} \mathrm{~s}^{-1}$ (extreme snapshot)] both far exceed the critical value $\left(\Delta U_{\text {critical }} \sim 0.2 \mathrm{~m} \mathrm{~s}^{-1}\right.$ taking $\beta_{\text {nd }}=0.05$ and $\left.F_{2}=0.25\right){ }^{4}$ Taken together with the Rayleigh condition test, this suggests that the KE jet is potentially subject to a mixed instability at this location.

A similar conclusion is reached by making a two-layer approximation and applying the two-layer model necessary condition for instability (Pedlosky 1963). The barotropic structure of the KE system below the thermocline suggests that this approximation of simplified vertical

\footnotetext{
${ }^{3}$ Here, $U$ is the zonal velocity; $\beta_{\text {nd }}$ is the nondimensional $\beta$ parameter, $\beta_{\text {nd }}=\beta L^{2} / U$, where $L$ and $U$ are typical length and velocity scales; and $F_{2}$ is the lower-layer Froude number, $F_{2}=f_{o}^{2} / g^{\prime} H_{2}$. Here, $f_{0}$ is the Coriolis parameter; $g^{\prime}$ is reduced gravity, $g^{\prime}=g\left(\rho_{1}-\rho_{2}\right) / \rho_{0}(g$ is the gravitational acceleration and $\rho_{1}, \rho_{2}, \rho_{0}$ are the density in the upper and lower layers and the reference density, respectively); and $H_{2}$ is the lower-layer depth.

${ }^{4}$ To compute appropriate nondimensional numbers, we take $U$ to be $1 \mathrm{~m} \mathrm{~s}^{-1}$ and $L$ to be $50 \mathrm{~km}$ as typical scales of the horizontal jet structure. Here, $F_{2}$ assumes a thermocline depth of $750 \mathrm{~m}$ in a 5000-m-depth ocean.
} 

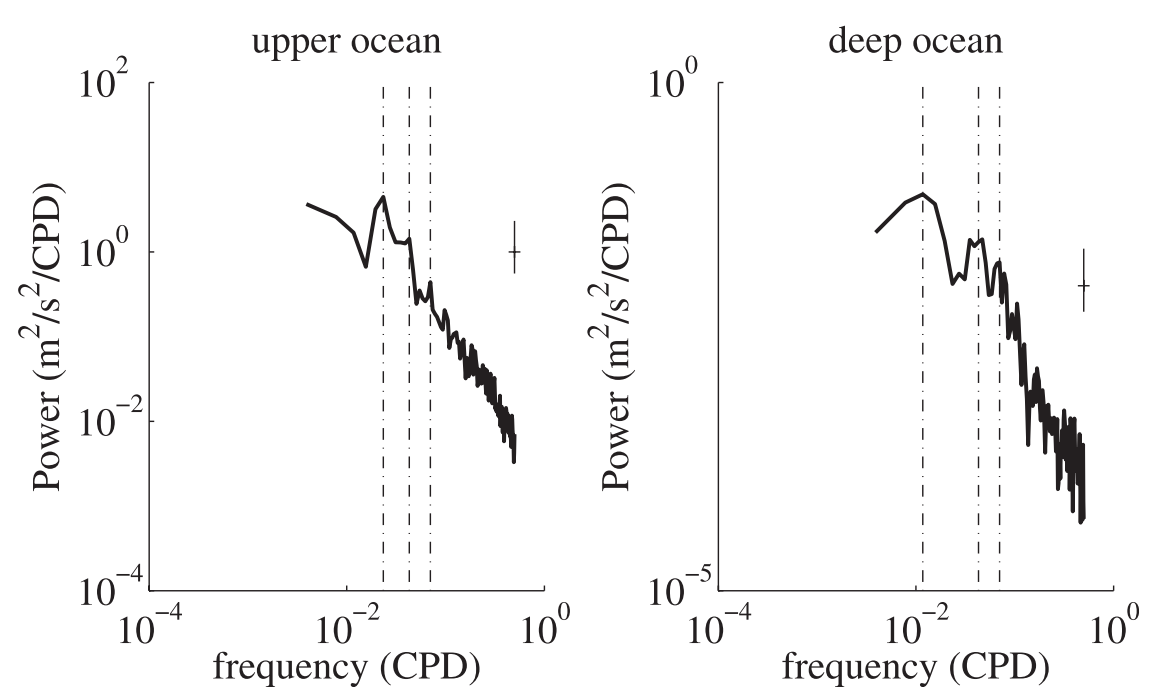

FIG. 15. An estimate of the power spectral density computed from the time series of alongstream velocity near the mean jet axis in the (left) upper and (right) deep ocean. Dashed-dotted lines indicate periods of (left) 40,20, and 16 days and (right) 85, 20, and 16 days. The height of the cross in the top right of each indicates the $95 \%$ confidence interval.

structure may be appropriate (Waterman 2009), and one can then evaluate the system's stability properties by examining the layered version of the meridional PV gradients (and whether a change of sign is observed in either the horizontal or the vertical). This is explored in Fig. 18. Here again, both the synoptic mean and extreme snapshot show that the layered versions of the vorticity gradient change sign in the vertical at the jet axis, confirming that the necessary condition for instability is satisfied. This is consistent with the results of Howe (2008), who determined the $\mathrm{PV}$ gradient associated with the mean stream-coordinate structure of the KE jet computed from the CPIES data for a subset of the KESS period and found it to change sign in both the horizontal and the vertical.

In short, both the synoptic mean and instantaneous snapshots of the horizontal and vertical shears observed during KESS indicate that shears can far exceed the critical values for both barotropic and baroclinic instability based on idealized Rayleigh and Phillips model criteria and for instability based on the meridional gradients of approximations to the layered PV gradients. Combined with the existence of enhanced energy at time scales predicted by appropriate linear stability calculations, this suggests that, even as far downstream as the KESS array

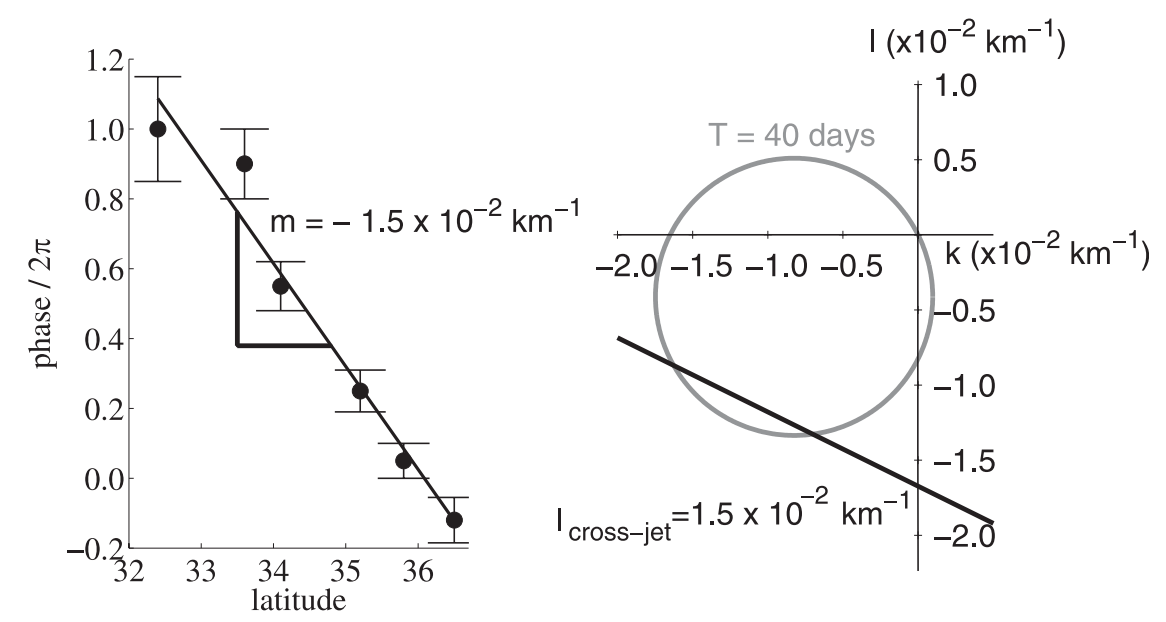

FIG. 16. (left) Phase vs latitude of the first EOF mode at 40-day period and (right) a test of the barotropic topographic Rossby wave dispersion relation for the observed wavenumber. 

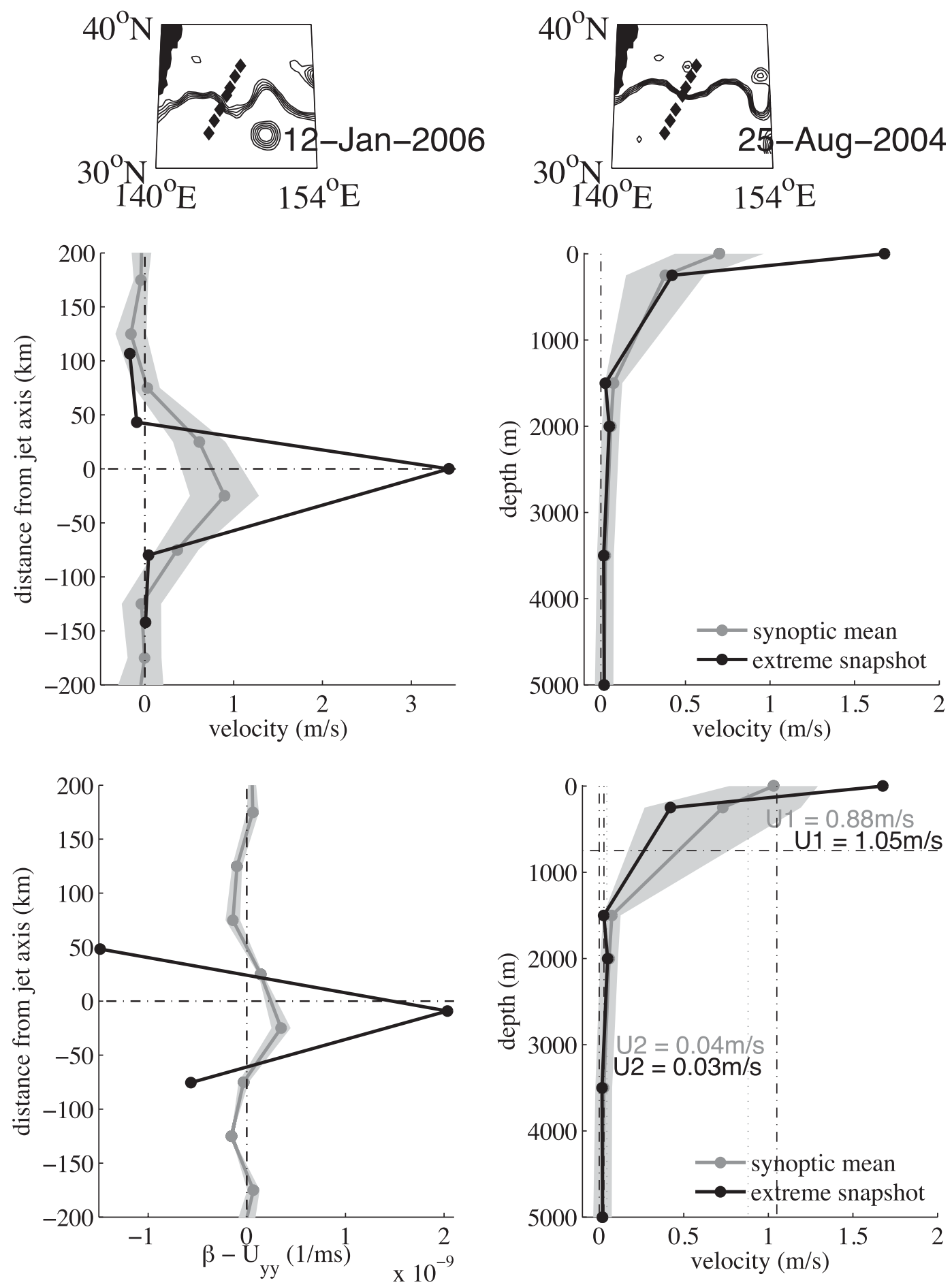

FIG. 17. An evaluation of the jet's potential for barotropic and baroclinic instability based on idealized Rayleigh and Phillips model criteria. (top) Synoptic mean (gray) and an extreme snapshot (black) of (left) the horizontal crossjet profile of along-stream velocity at 250-m depth and (right) the vertical profile of along-stream velocity at the jet axis to illustrate the jet velocity structure observed during KESS. (bottom) A consideration of the idealized (left) Rayleigh and (right) Phillips model necessary conditions for instability for the observed horizontal and vertical shears associated with the above velocity profiles. See the text for a full description. (top inserts) The state of the jet, as visualized by contours of the daily snapshot of SSH measured by altimetry, that correspond to the extreme snapshot below. Contours are in the range of $1.9-2.3 \mathrm{~m}$ in intervals of $0.1 \mathrm{~m}$. The date corresponding to each snapshot is as indicated. 

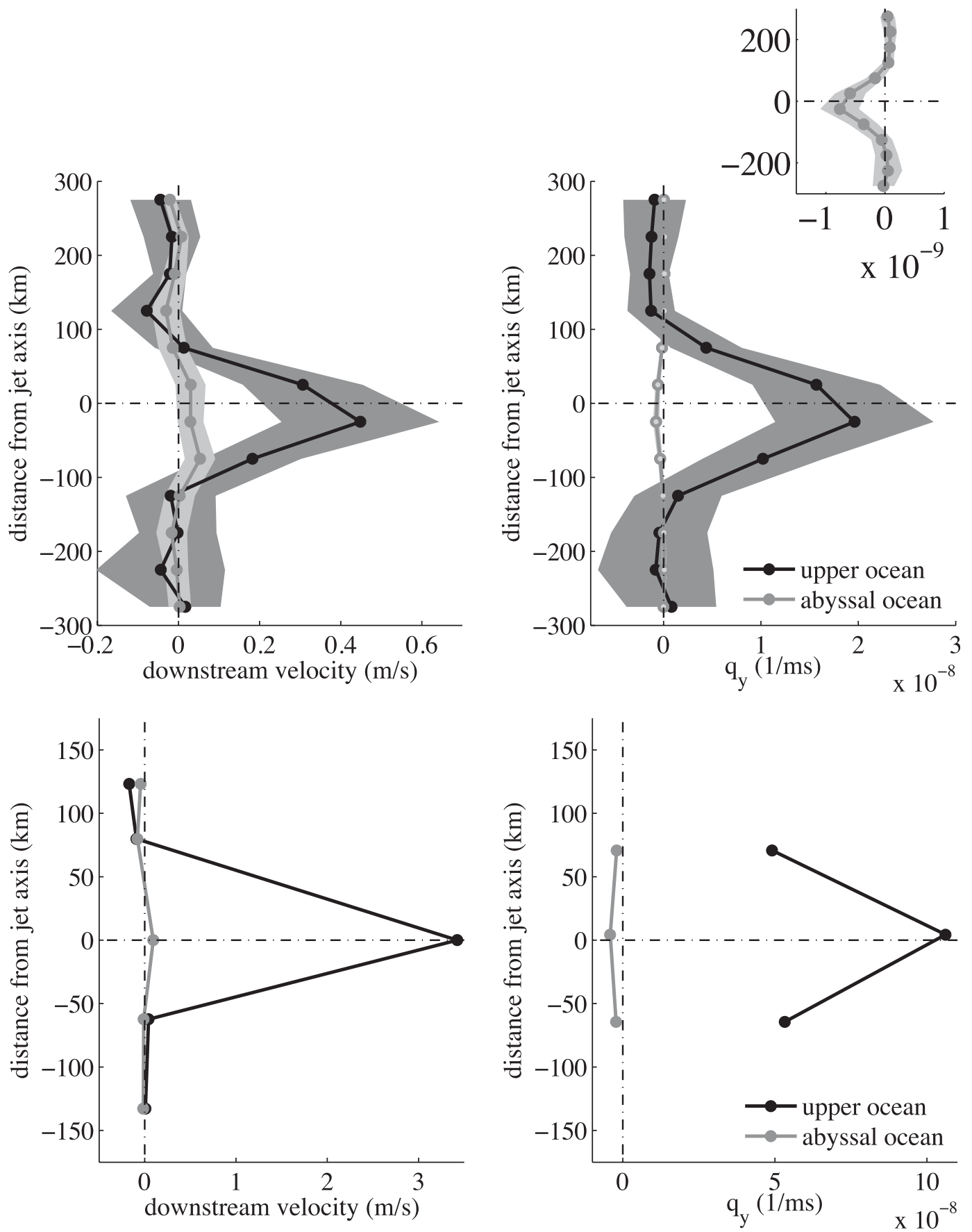

FIG. 18. An evaluation of the jet's potential for instability based on the two-layer model necessary condition for instability. (left) Approximations to the upper- and deep-ocean layer's horizontal cross-jet profiles of along-stream velocity for the (top) synoptic mean and (bottom) extreme snapshot as in Fig. 17. (right) Approximations to the upper- and deep-ocean layer's horizontal cross-jet profiles of the meridional vorticity gradient computed as $q_{1,2} y=$ $-u_{1,2 y y}+\beta \pm\left(f^{2} / N^{2} H_{n}^{2}\right)\left(u_{2}-u_{1}\right)$. Here $N$ is the buoyancy frequency, $H_{n}$ is the $n$th layer depth, and $f$ is the Coriolis frequency taken to be $5 \times 10^{-3} \mathrm{~s}^{-1}, 800 \mathrm{~m}(n=1)$ and $4000 \mathrm{~m}(n=2)$, and $1 \times 10^{-4} \mathrm{~s}^{-1}$ respectively. A close-up of the deep-ocean synoptic mean $q_{y}$ profile is shown in the top-right insert. 
location, the KE jet is potentially subject to a mixed instability mechanism that is a likely source of some of its eddy variability.

Again, we can approximate the relative importance of the potential instability at a 20-day period as a source of variance by comparing the EKE in the 20-day-period band to the total integrated EKE. The exercise shows that motions in this frequency band account for approximately $17 \%$ of the total variance in the upper ocean. In a similar way, the 16-day-period peak accounts for approximately $10 \%$ of the total upper-ocean variance. These are upper bounds for the contribution of instability because other sources could also contribute to the variability at these time scales. Instability modes at other frequencies may also significantly contribute to the total variance; however, it is difficult to quantify this as these motions are not identifiable from the spectra.

\section{Eddy-mean flow interactions}

Finally, we attempt to gain some insight into how the mean flow and eddy variability interact, with particular interest in evaluating the eddy effect on the mean. For some of the variability sources discussed in the previous section (viz., jet meandering and rings) it is possible to isolate the source and compare the mean jet structure with and without the source included. In this way, we see that jet meanderings acts to weaken and broaden the mean jet structure and that ring interactions (assuming their influence exists only during times when they are present) do not have a significant effect on the mean jet structure on the jet flanks. We note, however, that these crude tests of the eddy effect do not take into account dynamical changes to the system that result from the eddy source's action, and in this way these diagnostics do not tell the full story. Further, it is difficult to isolate the feedback effect of waves and jet instability on the mean jet-gyre system.

As a consequence, here we consider eddy-mean flow interactions in terms of dynamical quantities that give rise to eddy forcing of the mean: namely, the spatial gradients of the mean velocity variances and covariances or Reynolds stresses that give rise to the eddy flux divergences of momentum and vorticity that impact the time-mean flow. Even with the relatively high spatial and temporal resolution of the KESS mooring array, evaluating eddy forcing terms directly, which requires computing multiple zonal and meridional spatial derivatives of the variability fields, is not possible. We can, however, consider the cross-jet distributions of Reynolds stresses observed by the mooring array and explore their implications for eddy forcing and possible eddy-mean flow interaction scenarios. Here, we consider these
Reynolds stress distributions that are produced by the sum of all eddy sources and evaluate their summed implications for the eddy feedback effect. We also compare them to various simplified dynamical models of jet meandering, wave radiation, and jet instability in isolation to select the variability sources that could potentially dominate the net eddy-mean feedback effect in the KE. Finally, we compare observations of mean and eddy properties in the KE with the output of an idealized model of a WBC jet to test the relevance of our understanding of eddy-mean flow interactions in WBC jet systems derived from the model to the actual KE system.

\section{a. Eddy effect on the mean jet as diagnosed by $\partial \overline{u^{\prime} v^{\prime}} / \partial y$}

We first evaluate the cross-jet distribution of the timemean Reynolds stress $\overline{u^{\prime} v^{\prime}}$ (Fig. 19). The meridional gradient of this quantity has important implications for the feedback of eddies on the mean flow through its contribution to the eddy vorticity flux divergence, $-\partial^{2}\left(\overline{u^{\prime 2}}-\overline{v^{\prime 2}}\right) / \partial x \partial y+\partial^{2}\left(\overline{u^{\prime} v^{\prime}}\right) / \partial x^{2}-\partial^{2}\left(\overline{u^{\prime} v^{\prime}}\right) / \partial y^{2}$, and it is often exploited for its heuristic value in giving insight into an approximation of the effective eddy force on the mean flow given by the divergence of the $\mathrm{E}$ vector, $\left.\partial \overline{\left(v^{\prime 2}\right.}-\overline{u^{\prime 2}}\right) / \partial x-\partial\left(\overline{u^{\prime} v^{\prime}}\right) / \partial y$ (Hoskins et al. 1983). This latter diagnostic is valid as an approximation of the eddy force acting to accelerate the time-mean flow under conditions usually well satisfied in elongated storm tracks and WBC jets, and it is often dominated by the $\overline{u^{\prime} v^{\prime}}$ term in these systems where along-jet variations are small relative to cross-jet variations (for a complete discussion, see Hoskins et al. 1983). In the KESS mooring array measurements, a similar pattern of $\overline{u^{\prime} v^{\prime}}$ across the jet in both the upper and deep ocean is seen, with $\partial\left(\overline{u^{\prime} v^{\prime}}\right) / \partial y>$ 0 (implying a westward effective eddy force if one neglects the zonal gradient contribution) on the jet flanks and $\partial\left(\overline{u^{\prime} v^{\prime}}\right) / \partial y<0$ (implying an eastward effective eddy force making the same zonal gradient assumption as above) at the jet axis (Fig. 19). This is in the sense to accelerate the jet at its axis and accelerate the mean westward recirculations on the jet flanks, consistent with the hypotheses that eddies act to enhance the jet's transport and that the recirculations are, at least partially, eddy driven.

Note that this pattern of $\overline{u^{\prime} v^{\prime}}$ is not consistent with the scenario of a barotropically unstable jet, in which eddies flux momentum away from the jet and act to reduce its large-scale horizontal shear. Instead, this pattern mirrors that of a localized wave radiator, which, as discussed in Waterman and Jayne (2011, manuscript submitted to J. Phys. Oceanogr.), results from energy radiation from a localized source of wave or eddy activity. Both upperocean and deep-ocean patterns are thus consistent with the downstream wave-radiator regime in an along-stream 


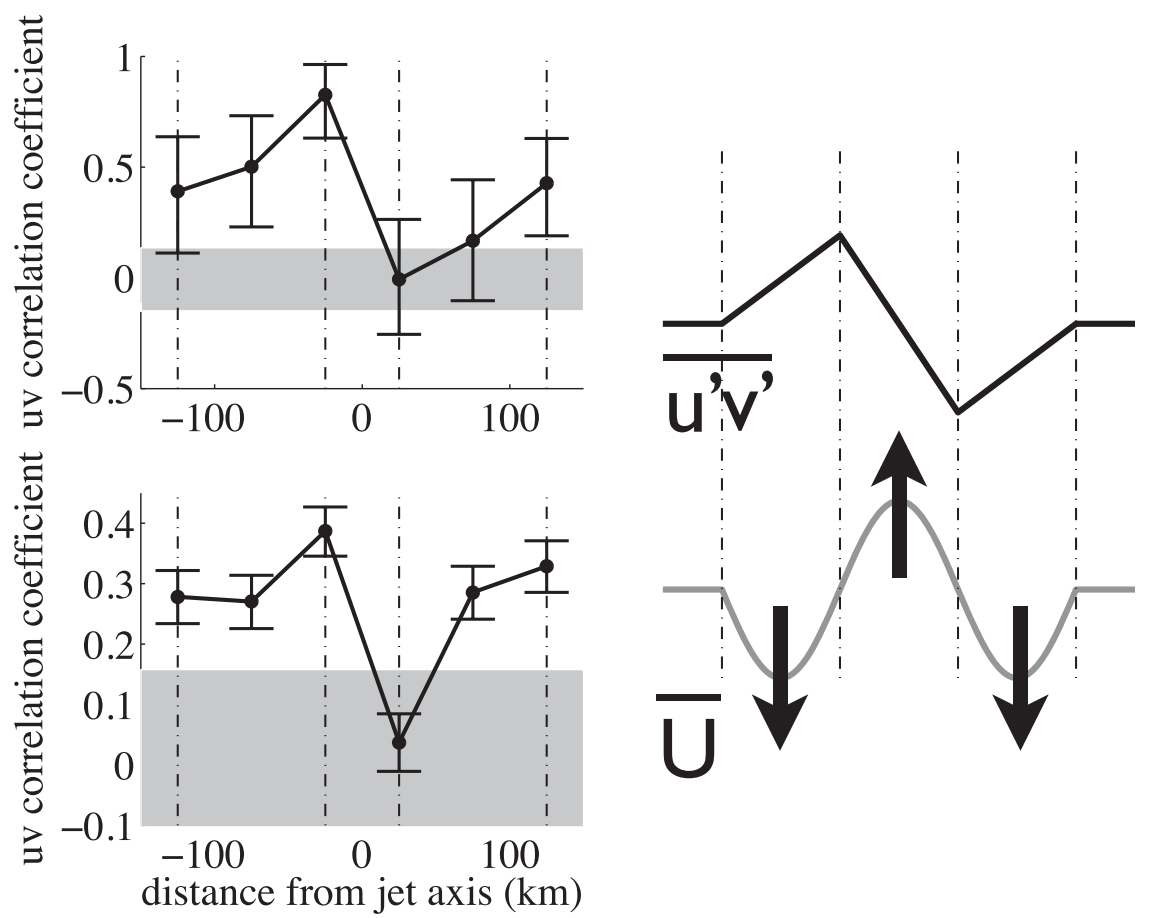

FIG. 19. (left) The cross-jet distribution of the mean correlation coefficient $\overline{u^{\prime} v^{\prime}}$ as measured by the KESS mooring array for the (top) upper and (bottom) deep ocean. (right) A schematic illustrating the sense of the effective eddy force derived from this $\overline{u^{\prime} v^{\prime}}$ pattern.

evolving mixed instability jet, as discussed in reference to an idealized WBC jet model by Waterman and Jayne (2011) (Fig. 20). The location of the KESS array near the position of the jet's maximum EKE (which in the idealized WBC model occurs close to the along-stream transition between the unstable-jet and the wave-radiator regimes) makes this suggestion of the KESS array belonging to the wave-radiator regime of the KE jet plausible. Important to an eddy-mean flow interaction perspective, as mentioned above, this is a pattern associated with the eddy driving of the mean recirculations.

\section{b. Plausible eddy-mean flow interaction scenarios}

To understand the relationship between the various sources of eddy variability discussed in section 4 and the eddy-mean flow interactions considered here we next consider the cross-jet distributions of all of the mean Reynolds stresses $\overline{u^{\prime} u^{\prime}}, \overline{v^{\prime} v^{\prime}}$, and $\overline{u^{\prime} v^{\prime}}$ as well as the timemean EKE structure observed by the KESS mooring array, and we compare them to those derived from models of various sources of variability relevant to the KE jet system in isolation. In this way, by looking for consistencies and discrepancies in the patterns, we hope to determine plausible and dominant eddy-mean flow interaction scenarios for the $\mathrm{KE}$ jet at this location that are consistent with the observed Reynolds stress distributions.
A comparison of the observed distributions and those of select idealized models is summarized in Fig. 21. Idealized models include the following (from simplest to most complex): 1) a simple meandering jet [a basic flow cosine jet with north and south walls in phase (the "sinuous mode") meandering past a line of fixed moorings]; 2) waves radiated from the "rigid corrugation model" (Hogg 1994) (the neutral Rossby wave field forced by a zonally oriented propagating boundary with sinusoidal corrugations); and 3) a barotropic unstable jet (Kamenkovich and Pedlosky 1996) (a basic state jet plus the perturbation field that arises from the linear stability analysis of a steady, barotropic unstable-jet profile; see the appendix for details).

The comparison shows that it is challenging to differentiate between different eddy-mean flow interaction scenarios because features of the Reynolds stress distributions of the various idealized models are either not unique or require fine cross-jet resolution to discern structure inside the width of the mean jet. Nevertheless, some general comments can be made:

- The single-peaked structure of $\overline{u^{\prime} u^{\prime}}$ observed in the upper ocean is consistent with the barotropically unstablejet model; the single-peaked structure of $\overline{v^{\prime} v^{\prime}}$, however, is not. 

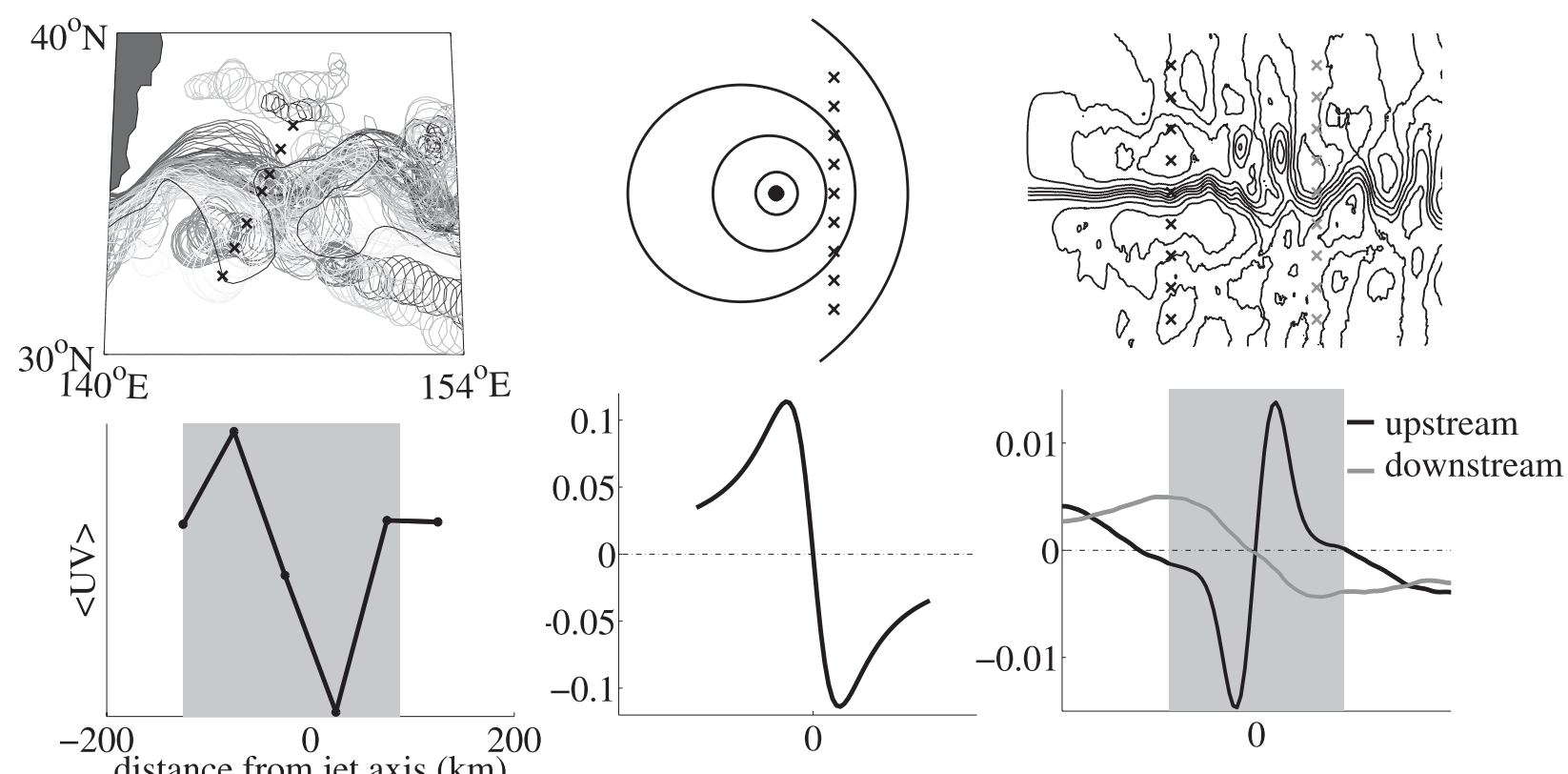

distance from jet axis $(\mathrm{km})$

FIG. 20. (top left) Observed KE jet variability as visualized by weekly snapshots of the 2.1-m SSH contour measured by satellite altimetry compared to two idealized models of variability sourced from the jet, (top middle) a localized wave radiator (lines indicate a snapshot of the instantaneous streamfunction of the Rossby wave field forced from a localized region denoted by the black circle), and (top right) an along-stream evolving mixed instability jet (again lines indicate a snapshot of the instantaneous streamfunction). In all, black Xs denote mooring locations (real and hypothetical). See the text for further details. (bottom left) The cross-jet distribution of $\overline{u^{\prime} v^{\prime}}$ observed in the deep ocean by the KESS mooring array compared to that for (bottom middle) the localized wave-radiator model and (bottom right) the along-stream evolving idealized jet. In the latter, upstream and downstream refer to the along-stream location of a transition from an unstable-jet regime to a wave-radiator regime as discussed in Waterman and Jayne (2011). The gray shading in all relevant subplots indicates the time-mean jet width.

- The double-peaked structure of $\overline{u^{\prime} u^{\prime}}, \overline{v^{\prime} v^{\prime}}$ and the mean EKE observed in the deep ocean is consistent with the rigid corrugation model (waves radiated from a meandering jet).

- The observed nonzero $\overline{u^{\prime} v^{\prime}}$ distribution indicates a scenario more complex than simply a meandering jet or the waves radiated from it. Jet instability or Rossby wave radiation from a localized source is consistent with the observation of a nonzero $\overline{u^{\prime} v^{\prime}}$ correlation.

In short, although the comparison of the observed crossjet Reynolds stress distributions with those of idealized models of eddy-mean flow interaction scenarios does not offer conclusive results, it does suggest the scenario of wave radiation from the deep meandering jet. The dominant scenario in the upper ocean remains unclear.

\section{c. Relevance of an idealized model of WBC jet eddy-mean flow interactions}

Finally, we view these new KESS results in the context of a broader description of eddy-mean flow interactions in the KE region and explore the potential relevance of our understanding of eddy-mean flow interactions in
WBC jet systems derived from study of an idealized model of a WBC jet (for a description of the model and the study's findings, see Waterman and Jayne 2011). In the model, an appreciation of zonal position relative to the along-stream location of the mean jet's stabilization, maximum in EKE, and maximum mean recirculation transport is fundamental to defining the nature of eddy-mean flow interactions. As a consequence, we examine KESS data in combination with in situ measurements from past programs upstream and downstream of KESS as well as satellite altimetry data in the $\mathrm{KE}$ region to characterize the along-stream development of both mean and eddy properties.

\section{1) Along-Stream eVolution of the MEAN JET-GYRE SYSTEM}

Using the combination of mooring array observations, we compare the along-stream evolution of the mean KE jet profile in both the upper and deep ocean to that of the mean jet in the upper and lower layers of the idealized model in a KE-like parameter regime and dimensionalized using the scales of the inflowing KE jet derived from satellite altimetry data (Fig. 22). Along-stream locations 

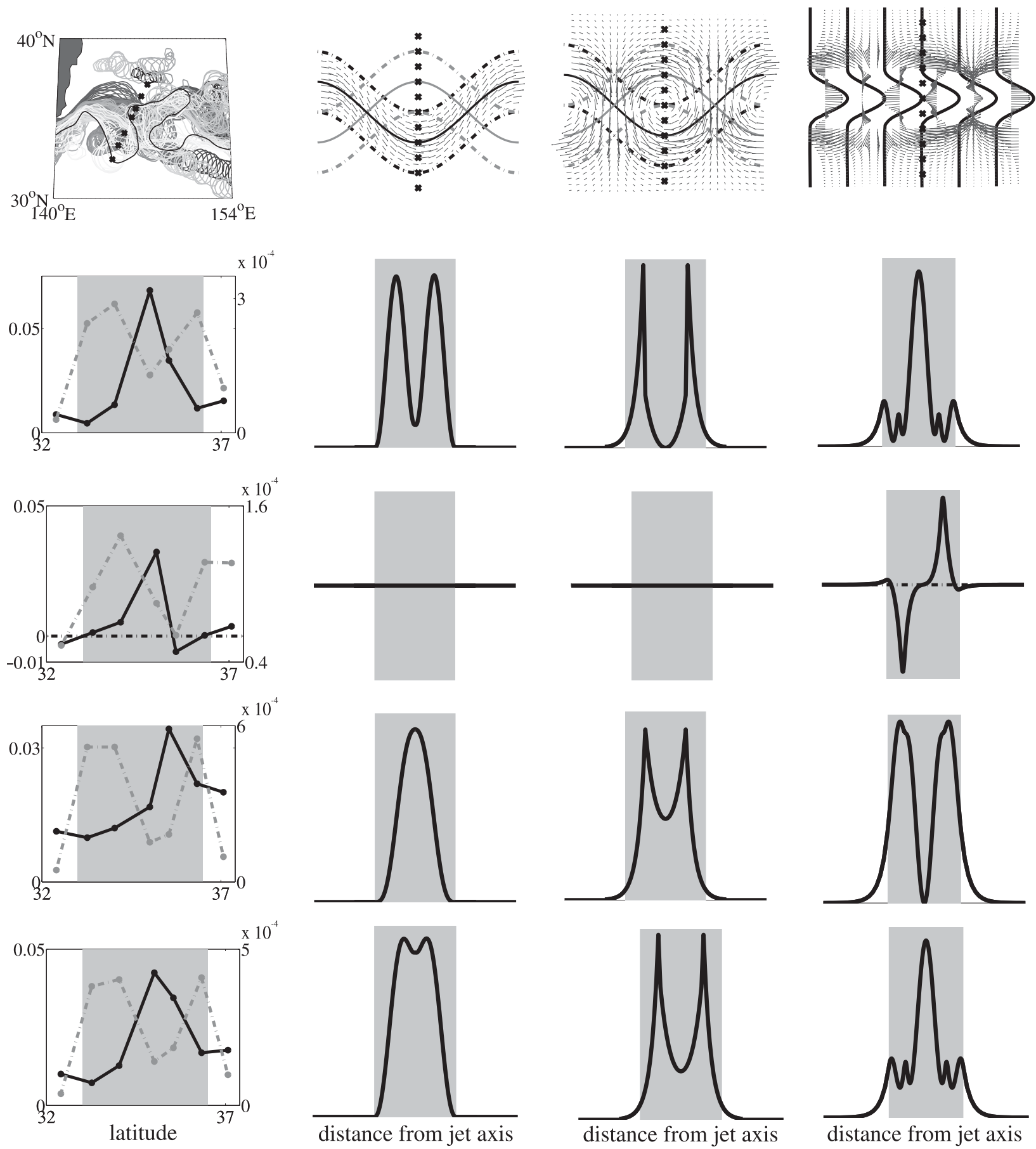

FIG. 21. (top) (left) Observed KE jet variability as visualized by weekly snapshots of the 2.1-m SSH contour measured by satellite altimetry compared to various idealized models of variability sourced from the jet, (top) (middle left) a meandering jet (gray and black solid lines indicate the jet axis and dash-dotted lines indicate the north and south walls of the jet in opposite phases of the meandering, vectors indicate the velocity inside the jet), (top) (middle right) the Rossby wave field generated by a meandering jet (as in the second column with vectors indicating the velocity of the radiated wave field), and (top) (right) a barotropically unstable jet (thick black lines indicate the meridional profile of the basic state jet, gray vectors indicate the velocity of the perturbation field). In all, black Xs denote mooring locations (real and hypothetical). The cross-jet distributions (in a stream-coordinate frame) of (second row) $\overline{u^{\prime} u^{\prime}}$, (third row) $\overline{u^{\prime} v^{\prime}}$, (fourth row) $\overline{v^{\prime} v^{\prime}}$, and (fifth row) $\overline{\mathrm{EKE}}=1 / 2\left(\overline{u^{\prime} u^{\prime}}+\overline{v^{\prime} v^{\prime}}\right.$ ) (left) observed by the KESS mooring array compared to those for the (second, third, and fourth columns) idealized models described above. Observed distributions are shown for the upper-ocean average (black) and deep-ocean average (gray) as defined in Fig. 11 with corresponding axes labels on the left and right, respectively. The gray shading in all relevant subplots indicates the time-mean jet width. 

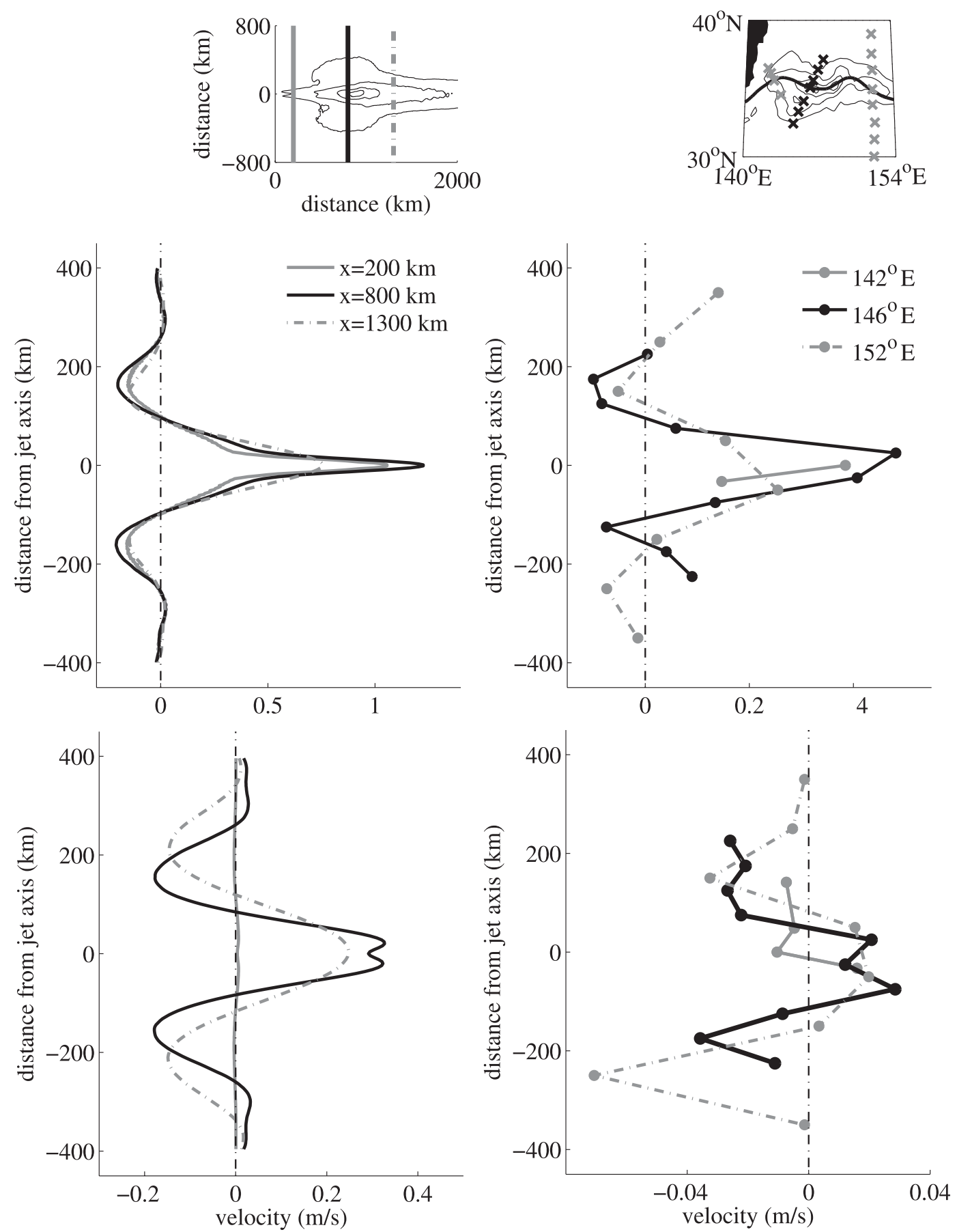

FIG. 22. A comparison of the along-stream evolution of the mean jet profile from an idealized model of a WBC jet in a KE-like regime vs that of the KE jet derived from mooring observations at three locations along stream. Cross-jet profiles of mean along-stream velocity for the (left) idealized model vs (right) mooring observations in the (top left) upper layer vs (top right) at 500-m-depth and (bottom left) lower layer vs (bottom right) deep-ocean average (average of all moorings deeper than the thermocline). Along-stream locations of the profiles relative to the mean EKE distribution (black contours) are indicated in the top inserts. 

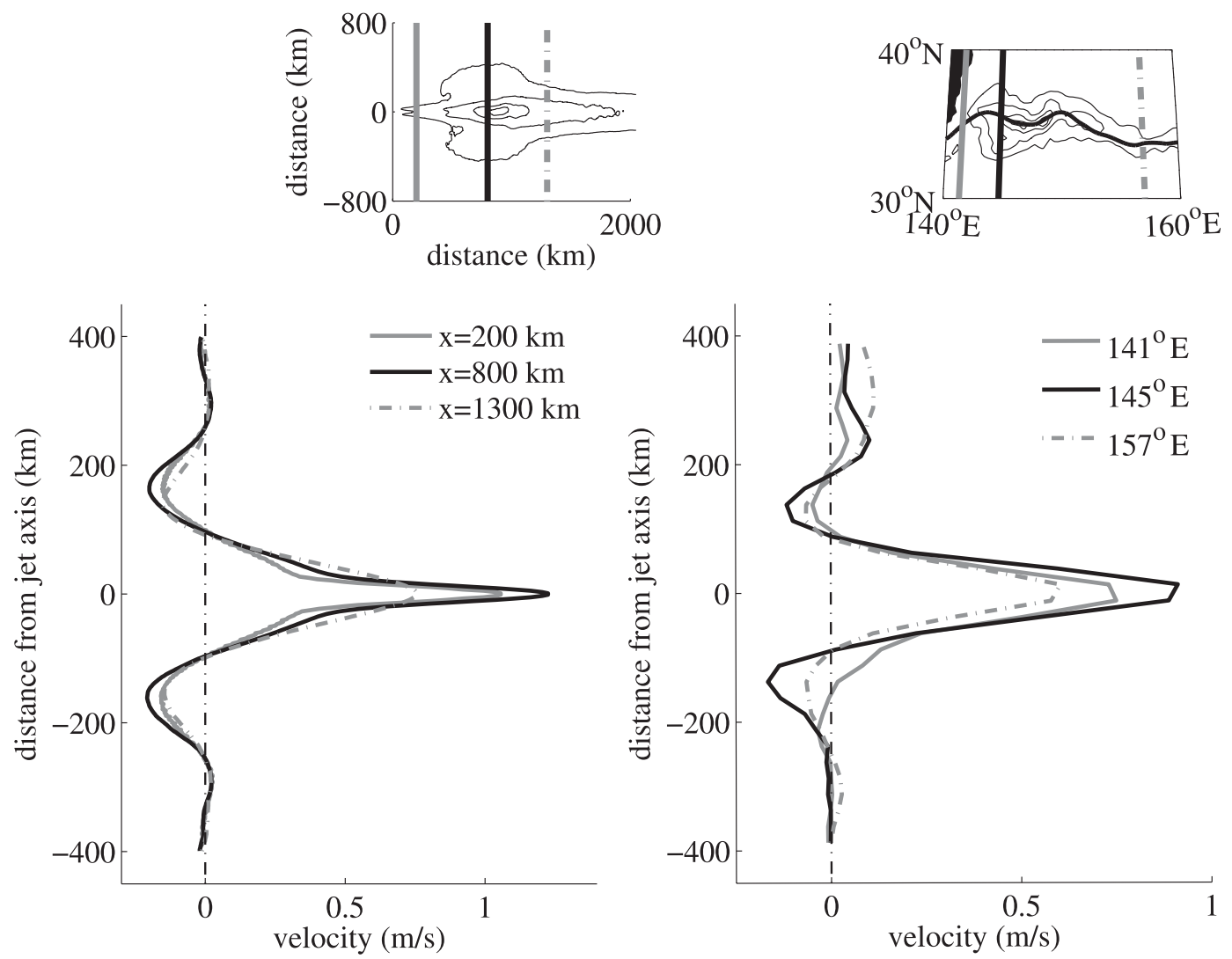

FIG. 23. As in Fig. 22, but with the stream-coordinate mean profiles of the KE jet derived from the 14-yr altimetry record replacing the mooring observations.

in the model for the comparison are selected to be similar to the mooring array locations relative to the time-mean EKE distribution (Fig. 22, top inserts). Note that the mean jet structure is viewed in the stream-coordinate frame, which is critical to revealing the details in the along-stream development of the jet-gyre structure. This is especially true for the case of the observations given the intense meandering of the KE jet and the coarse cross-jet resolution of the moorings. The comparison shows several important similarities between the model and the observations in the along-stream development of mean jet and gyre properties, including a strengthening of the upperlayer jet and the strengthening of westward recirculations up until the along-stream location of maximum EKE, followed by a weakening and broadening of the upperocean jet and a weakening of the recirculation strength downstream of the location of maximum EKE. This same along-stream development pattern is seen in the streamcoordinate mean profiles of the KE jet derived from the 14-yr altimetry record (Fig. 23). Here again, there is evidence of the jet and its flanking recirculations strengthening upstream of the EKE maximum and weakening downstream of this location, consistent with the model's behavior.

\section{2) AlOng-STREAm EVOLUTION OF PROPERTIES OF THE EDDY VARIABILITY AND SIGNATURES OF EDDY-MEAN FLOW INTERACTIONS}

Diagnosing the eddy effect on the mean is much more difficult using direct observations, but one insightful example of the observed along-stream evolution of eddy effect signatures is given in Fig. 24. Here, we compare the mean covariance ellipses (a visualization of the mean Reynolds stresses $\overline{u^{\prime} u^{\prime}}, \overline{v^{\prime} v^{\prime}}$, and $\overline{u^{\prime} v^{\prime}}$ ) computed from the mooring observations to those computed from the covariances diagnosed from the KE-like idealized model. Note that here it was found to be helpful to bandpass filter the observed velocity time series in a mesoscale frequency range (shown in Fig. 24 for a filter passing periods of 10100 days) to clarify the cross-jet patterns. Then important similarities in along-stream development are seen. In particular, we see a common, dynamically significant transition from a pattern of positive ellipse tilt north of the jet axis and negative ellipse tilt south of the jet axis in the nearfield of the jet (consistent with a barotropically unstable-jet regime) upstream of the location of maximum EKE to the reverse pattern (consistent with a wave-radiator regime) 

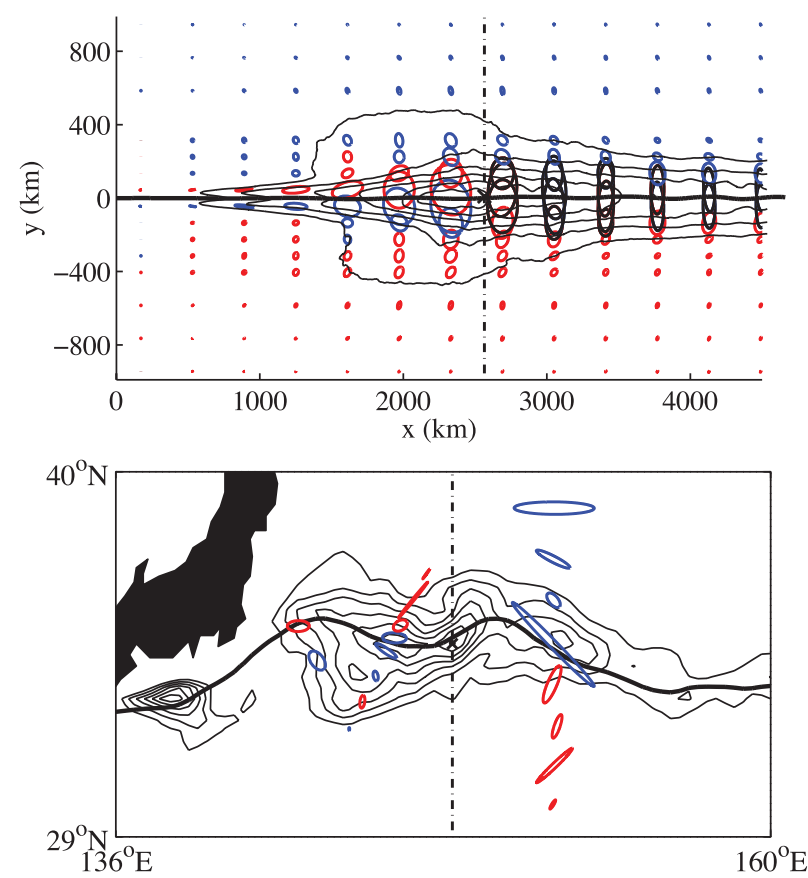

FIG. 24. A comparison of the mean covariance ellipses for (top) the layer-averaged fields in the two-layer model run in a KE-like regime vs (bottom) those for the KE jet as derived from the depthaveraged mooring observations.

downstream of this location. This suggests that there is potential relevance of the two-regime description of eddy effect on the mean found in the idealized WBC jet model to the along-stream development of the KE jet.

\section{Summary and discussion}

To summarize, using KESS mooring data, we present a characterization of the mean jet structure, the eddy variability, and the nature of their interactions relevant to the KESS location and during the KESS period.

We show important differences in the mean jet structure in the geographical versus stream-coordinate frame, including differences in mean jet strength, width, vertical structure, and flanking recirculation structure. Observations of the weakly depth-dependent recirculations to both the north and the south of the jet in the stream-coordinate frame provide, in combination with evidence of these recirculations seen in other forms of the KESS measurements (see Jayne et al. 2009), the first clear observational evidence of a northern recirculation gyre in the KE.

We categorize eddy variability at the KESS location according to some of its various sources: jet meandering, rings, waves, and instability. We describe their characteristics and estimate the relative contribution of each to the total variability. This evaluation shows them all to be significant sources of variability to the system. In the case of jet meandering and ring interactions, we are able to isolate these effects and thus evaluate the effect of these sources on the mean jet structure and its variability. In this way, we see that jet meandering acts to weaken and broaden the mean jet structure and that ring interactions (assuming their passage does not result in dynamical changes to the jet system) do not significantly impact the mean jet structure on the jet flanks.

Finally, we evaluate eddy-mean flow interactions by considering the spatial gradients of the Reynolds stresses that give rise to the eddy-mean feedback and evaluating their implications for eddy forcing. In this way, we provide evidence of an eddy effect that acts to enhance the mean jet's transport and drive the flanking recirculations. We attempt to link this eddy effect to the various sources of variability described above by comparing the observed Reynolds stress distributions to those of various idealized dynamical models of jet meandering, wave radiation, and instability and conclude that eddies act at the KESS location in the sense of a localized wave radiator. The comparison of KESS and additional regional observations with an idealized model of an along-stream evolving mixed instability jet suggests relevance of the model and the understanding of eddy-mean flow interactions in WBC jet systems derived from it to the KE system.

The results presented here are only the beginning of the observational analysis of eddy-mean flow interactions possible with the KESS data. We present evidence of a northern recirculation gyre and the suggestion that eddies may play a role in driving it, but the dynamics are still uncertain. Hence eddy-mean flow interactions in the KESS data should be quantified. Progress can be made by incorporating other forms of the KESS data (e.g., the MMP and CPIES measurements) and applying the methods of Bryden (1979) and Phillips and Rintoul (2000) to the pointwise MMP measurements and the methods of Cronin (1996) and Bower and Hogg (1996) to the two-dimensional data. This will allow the diagnosis of the eddy-mean energy and PV budgets and the characterization of the cases of upgradient and downgradient fluxes. The analysis of highresolution global circulation model output in this region would be useful as well, providing a larger temporal and spatial window into the $\mathrm{KE}$ and permitting statistical studies of the energy and PV fluxes. Interesting questions that could be addressed include the relative importance of relative vorticity versus thickness fluxes in the eddy forcing and the role of episodic events in the context of the timeaveraged observed fluxes.

There are also new open questions that are suggested by this initial look at the KESS data that would be interesting avenues to pursue in the future. We elaborate on two of particular interest below. 

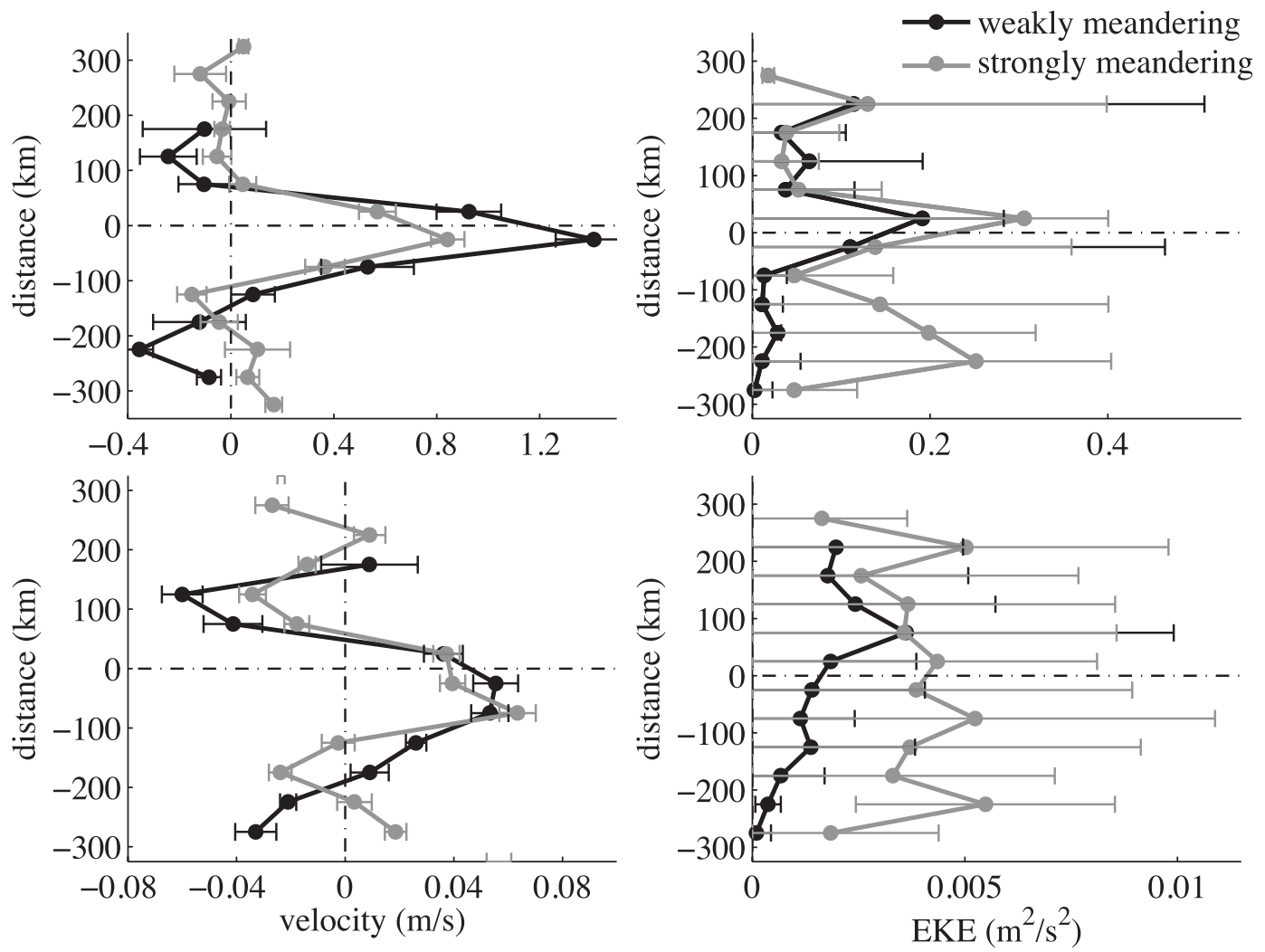

FIG. 25. A comparison of the (left) mean stream-coordinate jet structure and (right) EKE distribution computed over the time period in the KESS record during which the KE jet was in its weakly meandering state vs that when the KE jet was in its strongly meandering state. (top) The upper-ocean average and (bottom) the deep-ocean average (as defined in the caption of Fig. 11) are shown.

The first concerns the role of external features and influences in the jet-gyre system. Some of the largest waves, meanders, and ring formations in the KESS record appear to have been triggered by external features: deep synoptic eddies, warm and cold core rings, and other possibly winddriven eddies arriving from the east. Analogous to the jet stream, this observation contributes to the debate about whether a given synoptic disturbance in the jet system grows locally from infinitesimal perturbations or grows from the interaction with a preexisting finite amplitude feature. The relative fraction of mesoscale features that develop locally versus those that grow from external preexisting disturbances could be quantified in the KESS record. The source of many of these external features could be investigated.

The second topic that has arisen to be of particular interest during this study relates to the bimodal variability of the KE jet and the causes and mechanisms of its regime shifts. The KE jet is observed to exhibit a bimodality in its low-frequency behavior, oscillating between a quasi steady state consisting of quasi-stationary meanders and a highly variable state consisting of energetic meanders (the "stable" and "unstable" states, respectively) (Qiu and Chen
2005). Fortuitously, the KESS field program captured a regime transition in late 2004, with the most recent weakly meandering pattern, which had begun in 2001, switching to the strongly meandering state. ${ }^{5}$ Accompanying the shift in the state of the surface jet was also a dramatic change in the state of the subsurface mean jet and its eddy variability observed by the KESS array. For example, relative to the weakly meandering state, the strongly meandering state's mean jet became much weaker, especially in the upper ocean, and the recirculations became less prominent (Fig. 25, left). Note that these changes in the mean jet structure are observed in the stream-coordinate frame (i.e., with the smearing out effects of jet meandering removed), so this represents a real change in jet strength. As a consequence, both the horizontal and vertical shear in the

\footnotetext{
${ }^{5}$ We prefer the labels weakly meandering and strongly meandering as opposed to stable and unstable, because they do not make statements about the jet's hydrodynamic stability properties. As will be discussed, the two states of the KE jet are distinguished by changes in the meandering intensity of the jet, not necessarily the magnitudes of the jet shear and hence its hydrodynamic stability properties.
} 
TABLE A1. Time and length scales for various linear instability modes: barotropic jet.

\begin{tabular}{lllcc}
\hline \hline Mode & & & $\begin{array}{c}\text { Oscillation } \\
\text { period (days) }\end{array}$ & $\begin{array}{c}\text { Wavelength } \\
(\mathrm{km})\end{array}$ \\
\hline Zonal & Sinuous & Fastest growing & 25 & 300 \\
& Varicose & & 20 & 500 \\
Tilted & Sinuous & & 20 & 300 \\
& Varicose & & 20 & 500 \\
Zonal & Sinuous & Most radiating & 500 & 800 \\
& Varicose & & 400 & 1300 \\
Tilted & Sinuous & 400 & 800 \\
& Varicose & 200 & 1300 \\
\hline
\end{tabular}

strongly meandering period was much reduced relative to the weakly meandering state: that is, paradoxically the weakly meandering state jet looks much more unstable than the strongly meandering state based on the magnitudes of the velocity shears. Associated with the transition from the weakly meandering to strongly meandering state is also a dramatic increase in eddy energy, both in the upper ocean and also in the deep subthermocline ocean (Fig. 25, right). One hypothesis for the cause of the change in the dynamic state of the KE jet is that it arises from a change in the PV structure of the jet-gyre system (Spall 1996). This can be tested with the KESS data by examining the time evolution of the PV structure and its favorability for different instability modes in the different dynamical states during the KESS period. Insight into the question about whether changes leading to the rapid transition arise from processes generated locally or those that enter either from upstream or downstream can also potentially be gained from the KESS record. Comparing and contrasting the jet's structure, its eddy properties, and their interactions in the two states may provide new insight into how the transition occurs and what its implications are for the stability of the jet and the strength and extent of the recirculations. This may potentially resolve the paradox of the highly sheared jet showing low eddy variability and vice versa.

Acknowledgments. This work was supported by National Science Foundation funding for the KESS program under Grants OCE-0220161 (SW, NGH, and SRJ), OCE0825550 (SW), OCE-0850744 (NGH), and OCE-0849808 (SRJ). SW was also supported by the MIT Presidential Fellowship. The financial assistance of the Houghton Fund, the MIT Student Assistance Fund, and WHOI Academic Programs is also gratefully acknowledged. We thank the ships' crews of the R/V Thompson, R/V Revelle, and R/V Melville and the WHOI Subsurface Mooring Operations Group for their assistance in the KESS field program. We also thank Luc Rainville for processing the ADCP records and Maggie Cook for processing the VACM records. We gratefully acknowledge useful discussion with other
TABLE A2. Time and length scales for various linear instability modes: baroclinic jet.

\begin{tabular}{lllcc}
\hline \hline Mode & & & $\begin{array}{c}\text { Oscillation } \\
\text { period (days) }\end{array}$ & $\begin{array}{c}\text { Wavelength } \\
(\mathrm{km})\end{array}$ \\
\hline Zonal & Sinuous & Fastest growing & 5 & 100 \\
& Varicose & & 6 & 200 \\
Tilted & Sinuous & 5 & 100 \\
& Varicose & & 6 & 200 \\
Zonal & Sinuous & Most radiating & 30 & 200 \\
& Varicose & & 6 & 2000 \\
Tilted & Sinuous & 50 & 200 \\
& Varicose & 20 & 2000 \\
\hline
\end{tabular}

members of the KESS collaboration, in particular Luc Rainville, Andrew Greene, Kathleen Donohue, Randolph Watts, and Karen Tracey, as well as Joseph Pedlosky. More information and the observational data from KESS can be found online (http://uskess.org).

\section{APPENDIX}

\section{KE-Relevant Linear Stability Calculations}

We consider KE jet relevant linear stability calculations to compute the dynamical signatures (time scales, length scales, and cross-jet distributions of Reynolds stresses) that could potentially be associated with the instability of the KE jet. Comparing observed signatures to those predicted by these calculations is then a way to evaluate whether one sees evidence of potential jet instability. An outline of the calculations, the results, and their relation to the KESS observations are briefly presented here.

\section{a. Calculations}

We consider the perturbation field that arises from the linear instability of a steady, barotropically unstable-jet profile in one- and two-layer configurations following Kamenkovich and Pedlosky (1996). We solve the linearized quasigeostrophic potential vorticity equations for a specified basic state jet profile uniform in the along-jet direction given by $U(y)=U_{0}\left(1-y^{2}\right)^{3}$, where $U_{0}$ is the peak jet velocity at $y=0$ and $y$ is the meridional direction. The solution is obtained via a numerical "shooting technique" that varies the complex phase speed until the numerical solution in the interior matches the exterior analytic solution at the edge of the jet. In the barotropic case, this requires that the solution be continuous across the interface. In the two-layer case, continuity of the solution and its derivative is applied. The method gives the values of the phase speed $c$ (both real and imaginary), the meridional wavenumber $l$ (both real and imaginary), and the eigenfunction $\phi$ (the $y$-dependent part of the perturbation streamfunction) inside the jet. From this, the oscillation frequency $k c_{R}$ (where $k$ is the zonal wavenumber and $c_{R}$ is the real part of the complex 

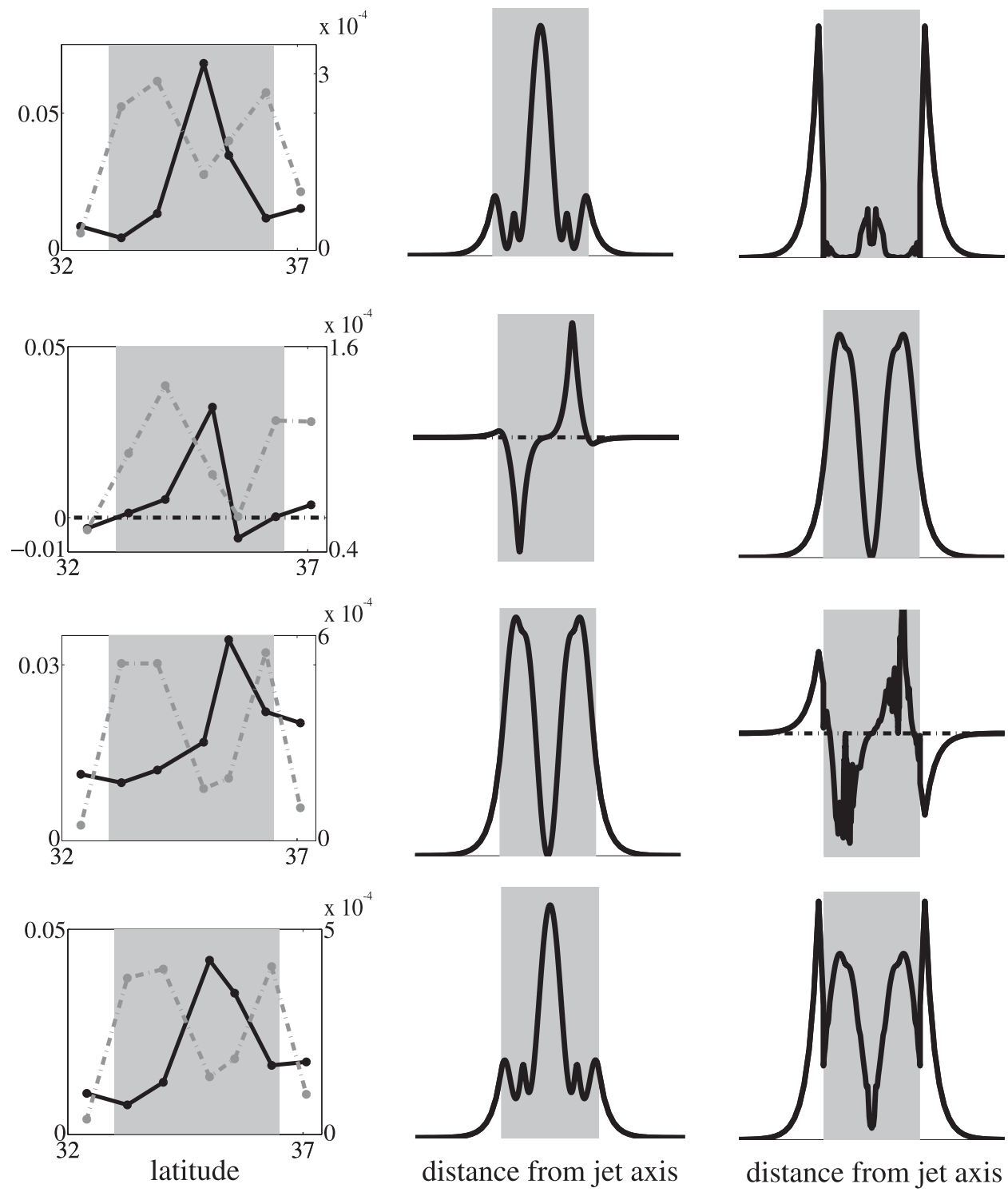

FIG. A1. The cross-jet distributions (in a stream-coordinate frame) of (first row) $\overline{u^{\prime} u^{\prime}}$, (second row) $\overline{u^{\prime} v^{\prime}}$, (third row) $\overline{v^{\prime} v^{\prime}}$, and (fourth row) $\overline{\mathrm{EKE}}=1 / 2\left(\overline{u^{\prime} u^{\prime}}+\overline{v^{\prime} v^{\prime}}\right)$ (left) observed by the KESS mooring array compared to those for the (middle) sinuous mode and (right) varicose mode from the linear stability calculation for a zonal, barotropic jet. Observed distributions are presented as in Fig. 21. The gray shading indicates the time-mean jet width.

phase speed) and the time-dependent velocity fields both inside and exterior to the jet can be computed.

We perform the calculation for a range of values of the zonal wavenumber $k$ and examine

- the different signatures of the sinuous versus varicose mode;

- the properties of the fastest growing and the most radiating mode;

- the effect of a meridional tilt to the orientation of the basic jet profile (of interest given the nonzonal nature of the mean KE jet at the KESS location as a consequence of the quasi-stationary meander); and

- the effect of baroclinicity.

\section{b. Results and their relation to the KESS} observations

\section{1) Time And Length SCALES}

The expected period of oscillation $T=2 \pi / k c_{R}$ and zonal wavelength $\lambda_{x}=2 \pi / k$ for a selection of calculations are given in Tables A1 and A2 for the barotropic 
and two-layer cases, respectively. We consider both the sinuous and varicose modes for a zonal jet and one tilted $30^{\circ}$ to the southeast (to model the orientation of the mean jet at the KESS location). We also consider both the properties of the fastest growing mode (i.e., the mode with the largest growth rate $k c_{i}$, where $c_{i}$ is the imaginary part of the complex wave speed) and the most radiating mode (the mode with the smallest ratio of meridional wavelength to meridional decay scale given by the ratio of the imaginary and real parts of the complex meridional wavenumber $l_{\mathrm{Im}} / l_{\mathrm{Re}}$ ).

In general, the calculations show that the varicose modes have universally larger zonal wavelengths (smaller $k$ ) than the corresponding sinuous modes and that the fastest growing modes have universally shorter periods of oscillation than the corresponding most radiating modes. Adding tilt does not change the zonal wavelength but can significantly alter the growth rate, radiating properties, period of oscillation, and the meridional wavelength and decay scales. Adding tilt tends to decrease the oscillation period, increase the meridional wavenumber (decrease the meridional wavelength), and increase the meridional decay scale at least in one direction. As a consequence, adding tilt makes both varicose and sinuous modes that were otherwise not strongly radiating (i.e., with a decay scale $1 / l_{\mathrm{Im}}$ shorter than the oscillation scale $1 / l_{\mathrm{Re}}$ so $l_{\mathrm{Im}} / l_{\mathrm{Re}}>1$ ) radiating (i.e., with a decay scale $1 / l_{\mathrm{Im}}$ longer than the oscillation scale $1 / l_{\mathrm{Re}}$ so $l_{\mathrm{Im}} / l_{\mathrm{Re}}<1$ ), consistent with the findings of Kamenkovich and Pedlosky (1996). Finally, adding baroclinicity (here in the form of a quiescent lower layer) also can significantly alter the time and length scales and radiation properties. Baroclinicity uniformly reduces the period and wavelength of all modes considered.

Interpreting the results in terms of their relevance to the scales of the KE jet allows us to highlight certain modes as consistent with the observed scales and hence select potential candidates for the instability experienced by the KE jet. Many of the modes considered do have oscillation periods on the order of the highestfrequency time scales observed as spectral peaks in the KESS velocity records. In particular, all of the fastest growing barotropic modes (sinuous and varicose; zonal and tilted) have oscillation periods that meet this criterion (i.e., periods on the order of 20-30 days). In general, the barotropic most radiating modes have periods much longer than those seen in observed spectra (400-500 days). Similarly, the barotropic fastest growing modes tend to have zonal wavelengths consistent with KE scales (300-500 km), whereas the most radiating modes tend to have unphysically long zonal wavelengths. Hence, these observations suggest a potential relevance of the barotropic fastest growing modes to the KE jet's instability while providing an argument against the relevance of the barotropic most radiating modes.

If one considers the baroclinic results, however, a different conclusion is reached. As a consequence of the uniform decrease in both oscillation period and zonal wavelength for all modes considered, with the addition of a second layer fastest growing modes now have a period that is significantly less than the 20-50-day mesoscale range, whereas the most radiating modes now have periods more consistent with the observed time scales. The wavelengths associated with the varicose most radiating modes remain unphysically large for KE scales, but the sinuous modes have physically reasonable length scales as well as time scales.

\section{2) VARIANCE STRUCTURE}

We also consider the meridional structure of the solutions because potential insight about relevant instability modes can also come from the comparison of the observed cross-jet distributions of Reynolds stresses and EKE with those predicted by the linear stability calculations. Here, we find that the structure of the perturbation field is not altered by whether the fastest growing or most radiating mode is considered, but rather it distinguishes between the sinuous versus varicose modes. Tilt plays an important role by introducing asymmetry between north and south of the jet, making both the meridional wavelength and decay scale different to the north versus to the south. Changing the meridional decay scale in particular introduces asymmetry into the magnitudes of the perturbation field north versus south of the jet, enhancing one flank's variance levels relative to the other.

Interpreting the results of solution structure in terms of their relevance to the KESS observations (Fig. A1), shows that the single-peaked structure of the variances observed in the upper ocean by the KESS moorings is suggestive of the sinuous mode, which is distinguishable from the varicose mode by its peak in $\overline{u^{\prime} u^{\prime}}$ and $\overline{\mathrm{EKE}}$ at the jet axis as opposed to on the jet edges. This is consistent with the conclusion of the baroclinic time and length scale calculations, and it offers further support for the hypothesis that the KE jet experiences a sinuous mode instability.

\section{REFERENCES}

Adamec, D., 1998: Modulation of the seasonal signal of the Kuroshio Extension during 1994 from satellite data.J. Geophys. Res., 103, 10 209-10 222.

Bower, A. S., and N. G. Hogg, 1996: Structure of the Gulf Stream and its recirculations at $55^{\circ}$ W. J. Phys. Oceanogr., 26, 1002-1022.

Bryden, H. L., 1979: Poleward heat flux and conversion of available potential energy in Drake Passage. J. Mar. Res., 37, 1-22. 
Cronin, M., 1996: Eddy-mean flow interaction in the Gulf Stream at $68^{\circ} \mathrm{W}$. Part II: Eddy forcing on the time-mean flow. J. Phys. Oceanogr., 26, 2132-2151.

Dewar, W. K., and J. M. Bane, 1989: Gulf Stream dynamics. Part II: Eddy energetics at $73^{\circ}$ W. J. Phys. Oceanogr., 19, 1574-1587.

Donohue, K., and Coauthors, 2008: Program studies the Kuroshio Extension. Eos, Trans. Amer. Geophys. Union, 89, doi:10.1029/ 2008EO170002.

— D. R. Watts, K. Tracey, A. D. Greene, and M. Kennelly, 2010: Mapping circulation in the Kuroshio Extension with an array of current and pressure recording inverted echo sounders. J. Atmos. Oceanic Technol., 27, 161-162.

Greene, A. D., 2010: Deep variability in the Kuroshio Extension. Ph.D. dissertation, Graduate School of Oceanography, University of Rhode Island, $150 \mathrm{pp}$.

Hall, M. M., 1986: Horizontal and vertical structure of the Gulf Stream velocity field at $68^{\circ} \mathrm{W}$. J. Phys. Oceanogr., 16, 1814 1828.

_ 1989: Velocity and transport structure of the Kuroshio Extension at $35^{\circ} \mathrm{N}, 152^{\circ}$ E. J. Geophys. Res., 94 (C10), 14 445-14 459.

- 1991: Energetics of the Kuroshio Extension at $35^{\circ} \mathrm{N}, 152^{\circ} \mathrm{E}$. J. Phys. Oceanogr., 21, 958-975.

Hallock, Z. R., and W. J. Teague, 1995: Current meter observations during the Kuroshio Extension Regional Experiment. Naval Research Laboratory Tech. Rep. MR/7332-95-7592, 119 pp.

Hogg, N. G., 1983: A note on the deep circulation of the western North Atlantic: Its nature and causes. Deep-Sea Res., 30, 945-961.

_ 1985: Evidence for baroclinic instability in the Gulf Stream recirculation. Prog. Oceanogr., 14, 209-229.

_- 1992: On the transport of the Gulf Stream between Cape Hatteras and the Grand Banks. J. Mar. Res., 50, 545-566.

1993: Toward parameterization of the eddy field near the Gulf Stream. Deep-Sea Res., 40, 2359-2376.

— 1994 : Observations of Gulf Stream meander-induced disturbances. J. Phys. Oceanogr., 24, 2534-2545.

— acoustic current meters. J. Phys. Oceanogr., 37, 148-161.

Hoskins, B. J., I. N. James, and G. H. White, 1983: The shape, propagation and mean-flow interaction of large-scale weather systems. J. Atmos. Sci., 40, 1595-1612.

Howden, S. D., 2000: The three-dimensional secondary circulation in developing Gulf Stream meanders. J. Phys. Oceanogr., 30, 888-915.

Howe, P. J., 2008: Stream-coordinate structure and variability of the Kuroshio Extension. M.S. thesis, Graduate School of Oceanography, University of Rhode Island, $123 \mathrm{pp}$.

Jayne, S. R., and Coauthors, 2009: The Kuroshio Extension and its recirculation gyres. Deep-Sea Res. I, 56, 2088-2099.
Kamenkovich, I. V., and J. Pedlosky, 1996: Radiating instability of nonzonal ocean currents. J. Phys. Oceanogr., 26, 622-643.

Pedlosky, J., 1963: Baroclinic instability in two-layer systems. Tellus, 15, 20-25.

Phillips, H. E., and S. R. Rintoul, 2000: Eddy variability and energetics from direct current measurements in the Antarctic Circumpolar Current south of Australia. J. Phys. Oceanogr., 30, 3050-3076.

Qiu, B., 1995: Variability and energetics of the Kuroshio Extension and its recirculation gyre from the first two-year TOPEX data. J. Phys. Oceanogr., 25, 1827-1842.

_ 2000: Interannual variability of the Kuroshio Extension system and its impact on the wintertime SST field. J. Phys. Oceanogr., 30, 1486-1502.

— recirculation gyre, and mesoscale eddies on decadal timescales. J. Phys. Oceanogr., 35, 2090-2103.

- , P. Hacker, S. Chen, K. A. Donohue, D. R. Watts, H. Mitsudera, N. G. Hogg, and S. R. Jayne, 2006: Observations of the subtropical mode water evolution from the Kuroshio Extension System Study. J. Phys. Oceanogr., 36, 457-473.

- S. Chen, P. Hacker, N. G. Hogg, S. R. Jayne, and H. Sasaki, 2008: The Kuroshio Extension northern recirculation gyre: Profiling float measurements and forcing mechanism. J. Phys. Oceanogr., 38, 1764-1779.

Schmitz, W. J., P. P. Niiler, R. L. Bernstein, and W. R. Holland, 1982: Recent long-term moored instrument observations in the western North Pacific. J. Geophys. Res., 87, 9425-9440.

Shay, T. J., J. M. Bane, D. R. Watts, and K. L. Tracey, 1995: Gulf Stream flow field and events near $68^{\circ} \mathrm{W}$. J. Geophys. Res., 100, 22 565-22 589.

Spall, M. A., 1996: Dynamics of the Gulf Stream/deep western boundary current crossover. Part II: Low-frequency internal oscillations. J. Phys. Oceanogr., 26, 2169-2182.

Thompson, R. O. R. Y., 1978: Reynolds stress and deep countercurrents near the Gulf Stream. J. Mar. Res., 36, 611-615.

Waterman, S., 2009: Eddy-mean flow interactions in western boundary current jets. Ph.D. thesis, Massachusetts Institute of Technology-Woods Hole Oceanographic Institution Joint Program, $264 \mathrm{pp}$.

—_, and S. R. Jayne, 2011: Eddy-mean flow interactions in the alongstream development of a western boundary current jet: An idealized model study. J. Phys. Oceanogr., 41, 682-707.

Watts, D. R., K. L. Tracey, J. M. Bane, and T. J. Shay, 1995: Gulf Stream path and thermocline structure near $74^{\circ} \mathrm{W}$ and $68^{\circ} \mathrm{W}$. J. Geophys. Res., 100, 18 291-18 312.

__ X. Qian, and K. L. Tracey, 2001: Mapping abyssal current and pressure fields under the meandering Gulf Stream. J. Atmos. Oceanic Technol., 18, 1052-1067. 